\title{
Applying double-magnetic induction to measure head-unrestrained gaze shifts: calibration and validation in monkey
}

\author{
Peter Bremen - Robert F. Van der Willigen • \\ Marc M. Van Wanrooij • David F. Schaling • \\ Marijn B. Martens • Tom J. Van Grootel • \\ A. John van Opstal
}

Received: 9 July 2010 / Accepted: 28 October 2010 / Published online: 17 November 2010 (C) The Author(s) 2010. This article is published with open access at Springerlink.com

\begin{abstract}
The double magnetic induction (DMI) method has successfully been used to record head-unrestrained gaze shifts in human subjects (Bremen et al., J Neurosci Methods 160:75-84, 2007a, J Neurophysiol, 98:3759-3769, 2007b). This method employs a small golden ring placed on the eye that, when positioned within oscillating magnetic fields, induces orientation-dependent voltages in a pickup coil in front of the eye. Here we develop and test a streamlined calibration routine for use with experimental animals, in particular, with monkeys. The calibration routine requires the animal solely to accurately follow visual targets presented at random locations in the visual field. Animals can readily learn this task. In addition, we use the fact that the pickup coil can be fixed rigidly and reproducibly on implants on the animal's skull. Therefore, accumulation of calibration data leads to increasing accuracy. As a first step, we simulated gaze shifts and the resulting DMI signals. Our simulations showed that the complex DMI signals can be effectively calibrated with the use of random target sequences, which elicit substan-
\end{abstract}

P. Bremen and R. F. Van der Willigen contributed equally to this work.

P. Bremen · R. F. Van der Willigen · M. M. Van Wanrooij .

M. B. Martens · T. J. Van Grootel · A. J. van Opstal ( $\varangle)$

Donders Institute for Brain, Cognition and Behaviour, Department

of Biophysics, Radboud University Nijmegen, Geert Grooteplein

21, 6525 EZ Nijmegen, The Netherlands

e-mail: j.vanopstal@donders.ru.nl

D. F. Schaling

Department of Ophthalmology, Radboud University Nijmegen Medical Center, Philips van Leydenlaan 15, 6525 EX Nijmegen,

The Netherlands

\section{P. Bremen}

Laboratory of Auditory Neurophysiology,

Department of Neurosciences, KU Leuven, O \& N II,

Herestraat 49, bus 1021, 3000 Leuven, Belgium tial decoupling of eye- and head orientations in a natural way. Subsequently, we tested our paradigm on three macaque monkeys. Our results show that the data for a successful calibration can be collected in a single recording session, in which the monkey makes about 1,500-2,000 goal-directed saccades. We obtained a resolution of 30 arc minutes (measurement range $[-60,+60]^{\circ}$ ). This resolution compares to the fixation resolution of the monkey's oculomotor system, and to the standard scleral search-coil method.

Keywords DMI $\cdot$ Search coil $\cdot$ Simulation .

Eye movements $\cdot$ Saccade $\cdot$ Macaca mulatta

\section{Introduction}

The double magnetic induction (DMI) method has been successfully applied to measure eye movements in headrestrained subjects and laboratory animals (Allik et al. 1981; Reulen and Bakker 1982; Bour et al. 1984; Ottes et al. 1987; Bos et al. 1988; Malpeli 1998; Zwiers et al. 2004). For this method, the subject's eye is positioned in the centre of two perpendicular (horizontal and vertical) oscillating primary magnetic fields that induce a superposition of ac-currents in a thin, golden ring that is inserted or implanted on the sclera of the eye. The currents in the ring in turn generate weak secondary magnetic fields that can be picked up by a coil placed closely in front of the eye. These secondary magnetic fields vary in a nonlinear way as a function of eye-in-head orientation, whereby the measurement range strongly depends on the exact geometrical relationships (i.e. size, distance, orientation and alignment) between ring and pickup coil (Bos et al. 1988; Bremen et al. 2007a). In addition, the pickup coil also carries the much stronger primary field components. This latter contribution of the primary fields can be reduced by 
adding a second coil that is connected in anti-phase to the pickup coil. If the subject's head is fixed, then this so-called anti-coil can be positioned within the primary fields in such a way that the primary-field cancellation is nearly complete (Bour et al. 1984; Ottes et al. 1987; Malpeli 1998). However, minor changes in geometry of the ring-coil/anti-coil assembly (e.g. through mechanical vibrations) can appreciably distort the measured signals. Moreover, when the head is unrestrained, cancellation will be far from perfect due to small electromagnetic and geometrical differences between pickup coil and anti-coil as well as to inhomogeneities of the primary fields, all resulting in head-orientation-dependent dc-offsets (Bremen et al. 2007a).

Yet, the major advantage of the DMI method when compared with the classical scleral search coil (SSC) technique (Robinson 1963; Collewijn et al. 1975) is the absence of vulnerable connecting wires from the eye. The ring is easily implanted in laboratory animals (DMI: Bour et al. 1984; SSC: Judge et al. 1979) and does not break. It is therefore also more comfortable to wear on the eye, e.g. of children and patients (Murphy et al. 2001; Irving et al. 2003). However, the restriction to head-restrained preparations, the necessity for a bite-board, the strong input-output nonlinearity, which, in combination with the limited measurement range, leads to ambiguities in the signals for angles exceeding about $20^{\circ}$, as well as the method's sensitivity to mechanical vibrations and artefacts have so far prevented its widespread acceptance.

Recently, we have shown that with some crucial modifications, the DMI method can be readily applied to measure twodimensional (2D) head-unrestrained gaze shifts in human subjects (Bremen et al. 2007b). We introduced two critical extensions to the classical head-restrained DMI method. First, addition of a third, frontal primary magnetic field, that is perpendicular to the horizontal and vertical fields disambiguates the nonlinear DMI signal. By combining the signals from all three fields with the associated head orientation (that can easily be measured e.g. with the search coil technique), the ring signal can in principle be calibrated over the full $360^{\circ}$ range. The second extension is calibration of the signals with artificial neural networks that make an analytical description of the complicated ring signals unnecessary. The networks account for the unknown inhomogeneities in the primary fields, for cross-talk as the fields are not exactly perpendicular, for the unknown ring/pickup coil geometry in 3D space, for additional position-dependent dc components that may be introduced by nearby equipment, as well as for the complex head-orientation-dependent dc-offsets that result from imperfect cancellation of primary field components.

In our previous study, we employed an intricate calibration routine in which human subjects were asked to generate a number of precisely coordinated eye-head movements (Bremen et al. 2007b). Although such an instructed calibration paradigm is feasible for adult humans, it is quite cumbersome (or even impossible) to apply to laboratory animals, as it would require extensive training. Our goal is to apply the DMI method to head-unrestrained laboratory animals, like cats, gerbils, chinchillas, marmosets and macaque and new world monkeys, while using a simple calibration routine. We propose and test a calibration paradigm that overcomes the forementioned problems, and demonstrate its applicability to gaze shifts made by three rhesus monkeys. The gaze-orienting task solely requires accurate fixation of randomly presented visual targets that are distributed over the range of interest. Such a paradigm can be readily learned by experimental animals, and is already used in numerous gaze-control studies.

\section{Simulations of the head-unrestrained DMI method}

In Sects. 2.2 and 2.3, we summarise the theoretical background of the DMI method for head-unrestrained gaze shifts in two dimensions. It will become clear that an analytical approach to calibrate real DMI signals is not feasible, as the 3D geometrical and electromagnetic properties of the DMI system are very difficult, if not impossible, to assess with sufficient precision. We therefore resorted to a black-box approach of the calibration routine, in which artificial neural networks were trained to map the set of input signals to known target locations. As there exists no a priori way to determine the appropriate network architecture that is capable of calibrating all possible eye-head combinations underlying a given gaze angle, we first explored the problem through extensive numerical simulations. The simulations embedded the (approximate) physics of the DMI method (described in Sects. 2.2 and 2.3) to produce voltages of the ring-coil assembly as function of eye-head orientations in space, as well as a simplified gaze-control model that generated a rich-enough repertoire of realistic eye-head saccadic gaze shifts with the appropriate variability of eye-head coupling, and eye-head kinematics (described in Sect. 2.1). The aim of the simulations was to determine the minimum data set and network architecture to produce adequate calibration accuracy across the entire measurement range.

\subsection{Simulations of eye-head gaze shifts}

The purpose of the simulations was to create a simple tool that allowed quantitative assessment of different potential calibration paradigms for later use in the experiments with real subjects. We implemented a simplified 2D version of Robinson's local feedback model (Van Gisbergen et al. 1981; Van Wetter and Van Opstal 2008) that could simulate pseudo-realistic head- and eye saccades for which the main parameters were randomly varied over a wide range: eyeand head saccade-component kinematics, initial eye-in-head 
Table 1 Parameters for gaze shift and head saccade generation

\begin{tabular}{|c|c|c|}
\hline Parameter & Value & Description \\
\hline$t$ & 400 & Trial duration in samples \\
\hline$n$ & & Gaussian noise with $\mu=0^{\circ}$ and $\sigma=0.05^{\circ}$ \\
\hline$G_{\mathrm{h}} V_{\mathrm{pk}}$ & 600 & Horizontal gaze peak velocity in deg/sample of the BG \\
\hline$G_{\mathrm{v}} V_{\mathrm{pk}}$ & 800 & Vertical gaze peak velocity in deg/sample of the BG \\
\hline$G m_{0}$ & 7 & Time to rise to $70 \%$ of peak velocity in sample of the BG \\
\hline$G T_{1}$ & 150 & Long time constant of the oculomotor plant $\rightarrow$ elastic properties of the muscles \\
\hline$G_{\text {on }}$ & 80 & Gaze shift onset re. begin of trial in samples \\
\hline$H_{\mathrm{h}} V_{\mathrm{pk}}$ & 400 & Horizontal head peak velocity in deg/sample of the BG \\
\hline$H_{\mathrm{v}} V_{\mathrm{pk}}$ & 300 & Horizontal head peak velocity in deg/sample of the BG \\
\hline$H m_{0}$ & 15 & Time to rise to $70 \%$ of peak velocity in sample of the BG \\
\hline$H T_{1}$ & 300 & Long time constant of the head plant $\rightarrow$ elastic properties of the muscles \\
\hline$H_{\mathrm{on}}$ & 20 & Head saccade onset re. gaze onset in samples \\
\hline$H_{\text {gain }}$ & $0.5-1$ & Head gain drawn from a Gaussian distribution with $\mu=0.75$ and $\sigma=0.05$ \\
\hline$T_{2}$ & 20 & Short time constant of oculomotor and head plant $\rightarrow$ plant's viscosity \\
\hline
\end{tabular}

position, and the head-saccade gain (head amplitude divided by desired gaze amplitude). We generated the horizontal and vertical saccade components independently to create various amounts of curvature in the gaze- and head trajectories. We also added different amounts of white noise to the simulated signals to test robustness.

Parameters of the horizontal and vertical burst generators (BGs) differed for eye and head (see Table 1). We added random noise to these parameters to allow for a substantial amount of inter-trial variability, both in eye-head coupling, as well as in eye-head kinematics and trajectories. Here, only one basic module of the 2D eye-head system (consisting of independent horizontal and vertical eye, and horizontal and vertical head modules) is described.

\subsubsection{Details of the gaze-shift simulations}

We begin by defining the instantaneous motor error of the module $m_{\mathrm{e}}(t)$ as

$m_{\mathrm{e}}(t)=L-S(t)$

with $S(t)$ the instantaneous saccade displacement evoked by a step of the target location, $L$. The brainstem BG encodes saccade velocity $v(t)$ by the following saturating nonlinearity:

$v(t) \equiv \frac{\mathrm{d} S(t)}{\mathrm{d} t}=v_{\text {peak }} \cdot\left(1-\exp \left(-m_{\mathrm{e}}(t) / m_{0}\right)\right)$

This BG contains two free parameters: the angular constant $m_{\mathrm{o}}$ (in deg) and the asymptotic peak velocity, $v_{\text {peak }}$ (in deg/s). Its output is fed to the neural integrator, which represents eyeor head position $S(t)$ (found by integrating Eq. 2 and solving for $S(t))$ :

$$
\begin{array}{r}
S(t)=m_{0} \cdot \ln \left(\frac{A \cdot \exp \left(v_{\text {peak }} t / m_{0}\right)}{1+A \cdot \exp \left(v_{\text {peak }} t / m_{0}\right)}\right), \\
\text { where } A \equiv \frac{1}{1-\exp \left(-L / m_{0}\right)}
\end{array}
$$

This signal is added to the scaled burst from the $\mathrm{BG}\left(T_{1} \cdot v(t)\right)$, and the resulting motoneuron pulse-step signal is subsequently convolved with the eye-, or head-motor plant impulse response:

$h(t)=\frac{1}{T_{1}-T_{2}}\left(\exp \left(-t / T_{1}\right)-\exp \left(-t / T_{2}\right)\right)$

with $T_{1}$ the long time constant (elastic properties of the plant) and $T_{2}$ the short time constant (related to plant viscosity). Note that since we simulated vertical and horizontal components independently, oblique saccades did not exhibit component stretching (e.g. Van Gisbergen et al. 1985). This detail, however, is not essential for the purpose of this study. To create realistic interactions between eye and head, and to account for subject idiosyncrasy, we randomly varied the head-saccade gain between 0.5 and 1.0 from trial to trial (Fuller 1992). At the same time, all the generated saccades obeyed the restriction that the eye-in-head orientation should not exceed $\pm 30^{\circ}$ in azimuth and elevation because of physical limitations (oculomotor range). To better mimic real experimental data, we added Gaussian noise $n$ with $\mu=0^{\circ}$ and $\sigma=0.05^{\circ}$ to each simulated sample. Note that experimentally measured SDs of gaze- and head-fixation noise were only $0.03^{\circ}$ and $0.02^{\circ}$, respectively. Table 1 lists the values of all parameters for the simulated eye-head gaze shifts.

To run the same series of trials as in the experimental setup with the monkeys (see Sect. 2.6), we simulated eye-head gaze shifts to random target locations distributed between 
$\alpha= \pm 80^{\circ}$ and $\varepsilon=-55$ to $85^{\circ}$ (see Sect. 2.4). Fixation and target locations were chosen such that their difference did not exceed $\pm 50^{\circ}$ in $\alpha$ and $\varepsilon$ since the field of view of a subject wearing the DMI assembly was restricted to about this range. Each simulated trial had a duration of 400 samples and started with a fixation epoch followed by a target epoch between sample numbers 80 and 400 .

\subsection{Computational analysis of simulated head-unrestrained DMI signals}

\subsubsection{The idealised situation}

This section summarises the physics of the double-magnetic induction (DMI) method. For further details, the reader is referred to Bos et al. (1988), and Bremen et al. (2007a). The physical analysis is described for the idealised situation in which the ring on the eye (radius $R_{\text {ring }}$ ) and the pickup coil in front of the eye (number of turns $N_{\text {pick }}$, radius $R_{\text {coil }}$ ) are exactly aligned in the $(\mathrm{H}, \mathrm{V})$ plane, with their normal vectors aligned when the eye is looking straight ahead, and intersecting in the same point of origin when the eye rotates. The distance between the aligned centres of ring and coil is $d \mathrm{~cm}$. Both are positioned within three homogeneous and mutually perpendicular oscillating magnetic fields (horizontal $(\mathrm{H})$, vertical $(\mathrm{V})$ and frontal $(\mathrm{F})$ ), with angular frequencies $\omega_{\mathrm{H}}, \omega_{\mathrm{V}}$ and $\omega_{\mathrm{F}} \mathrm{rad} / \mathrm{s}$ and strengths $B_{\mathrm{H}}, B_{\mathrm{V}}$ and $B_{\mathrm{F}}$, respectively. The magnetic fields induce alternating currents in the ring, which depend on its orientation within the fields. In the head-fixed case, the pickup coil is fixed in the $(\mathrm{H}, \mathrm{V})$ plane (and hence no flux from the $\mathrm{H}, \mathrm{V}$ fields), while the eye (radius $\left.R_{\text {eye }}\right)$ can rotate around its fixed centre in horizontal (angle $\alpha$ ) or vertical (angle $\varepsilon$ ) directions.

In the above mentioned case, it follows from the BiotSavart law that when the eye is rotated by $\alpha$ deg with regards to straight ahead, the voltage induced in the pickup coil by the induction current in the ring from the horizontal magnetic field is given by Bos et al. (1988); Bremen et al. (2007a):

$V_{\mathrm{H}}(\alpha, t)=K_{\mathrm{H}}(t) \cdot \sin (\alpha) \cdot L[\cos (\alpha)]$

where $K_{\mathrm{H}}(t)$ is a proportionality constant that only depends on the geometry of the system and time-varying magnetic field strength, $\sin (\alpha)$ the strength of the magnetic flux through the ring as function of horizontal eye rotation, and $L[\cos (\alpha)]$ the so-called shape factor that expresses the geometrical nonlinearity of the DMI method (see below). The coefficient $K_{\mathrm{H}}(t)$ can be expressed as

$$
\begin{aligned}
K_{\mathrm{H}}(t)= & N_{\text {coil }} \cdot \omega_{\mathrm{H}}^{2} \cdot B_{\mathrm{H}} \cdot \sin \left(\omega_{\mathrm{H}} t\right) \\
& \times \frac{\pi^{2} \cdot \mu_{\mathrm{o}} \cdot R_{\text {ring }}^{3} \cdot R_{\text {coil }}}{b \cdot Z_{\text {elec }}} \text { with } \\
b= & \sqrt{\left(R_{\text {eye }}+d\right)^{2}+R_{\text {coil }}^{2}}
\end{aligned}
$$

with $\mu$ the magnetic permeability, and $Z_{\text {elec }}$ the electrical impedance of the ring.

The shape factor distinguishes the DMI method from the scleral search-coil technique by introducing an additional nonlinearity that limits the measurement range to about $\pm 30^{\circ}$. It expresses the geometric dependence of the ring and the pickup coil by (Bos et al. 1988):

$$
\begin{aligned}
L[\cos (\alpha)]= & \sum_{n=1}^{\infty} \frac{1}{n(n+1)} \cdot\left(\frac{c}{b}\right)^{n} \cdot P_{n}^{1}\left(\frac{R_{\text {eye }}}{c}\right) \\
& \times P_{n}^{1}\left(\frac{R_{\text {eye }}+d}{b}\right) \cdot P_{n}(\cos (\alpha)) \text { with } \\
c= & \sqrt{R_{\text {eye }}^{2}+R_{\text {ring }}^{2}}
\end{aligned}
$$

Here, $P_{n}$ are Legendre polynomials and $P_{n}^{1}$ are associated Legendre polynomials of the first kind (about six terms suffice for an approximation within $0.05 \%$ ).

A similar set of equations holds for the vertical magnetic field and vertical eye angle $\varepsilon$. For the frontal field the magnetic flux is proportional to $\cos (\alpha)$ :

$V_{\mathrm{F}}(\alpha, t)=K_{\mathrm{F}}(t) \cdot \cos (\alpha) \cdot L[\cos (\alpha)]$

Note that the $L[\cos (\alpha)]$ term is only determined by the geometry of the coil-ring assembly, and does not depend on the orientation of the assembly with respect to the magnetic fields. In contrast, the $\sin (\alpha)$ and $\cos (\alpha)$ factors are fully determined by the primary magnetic flux through the ring. Thus, in the situation that the entire ring-pickup coil assembly of Fig. 1 is rotated by a horizontal gaze (eye-in-space) angle with respect to the magnetic fields, $g=\alpha+\gamma$ (with $\gamma$ the horizontal head angle), only the flux-related terms in Eqs. 5 and 8 will change and the induction voltages become

$$
\begin{aligned}
& V_{\mathrm{H}}(\alpha, \gamma, t)=K_{\mathrm{H}}(t) \cdot \sin (\alpha+\gamma) \cdot L[\cos (\alpha)] \\
& V_{\mathrm{V}}(\alpha, \gamma, t)=0 \\
& V_{\mathrm{F}}(\alpha, \gamma, t)=K_{\mathrm{F}}(t) \cdot \cos (\alpha+\gamma) \cdot L[\cos (\alpha)]
\end{aligned}
$$

Pure vertical rotation angles yield a similar set of equations (with $V_{\mathrm{H}}(\varepsilon, \delta, t)=0$ ). For combined horizontal/vertical rotations only the frontal field is sensitive to changes in both directions, so that in good approximation:

$$
\begin{aligned}
V_{\mathrm{H}}(\alpha, \gamma, \varepsilon, \delta, t)= & K_{\mathrm{H}}(t) \cdot \sin (\alpha+\gamma) \cdot L[\cos (\alpha)] \\
V_{\mathrm{V}}(\alpha, \gamma, \varepsilon, \delta, t)= & K_{\mathrm{V}}(t) \cdot \sin (\varepsilon+\delta) \cdot L[\cos (\varepsilon)] \\
V_{\mathrm{F}}(\alpha, \gamma, \varepsilon, \delta, t)= & K_{\mathrm{F}}(t) \cdot \cos \left(\sqrt{(\alpha+\gamma)^{2}+(\varepsilon+\delta)^{2}}\right) \\
& \times L[\cos (\alpha)] \cdot L[\cos (\varepsilon)]
\end{aligned}
$$

\subsubsection{The real situation}

It is important to note that Eq. 10 assume that the signals in the pickup coil only arise from the secondary 


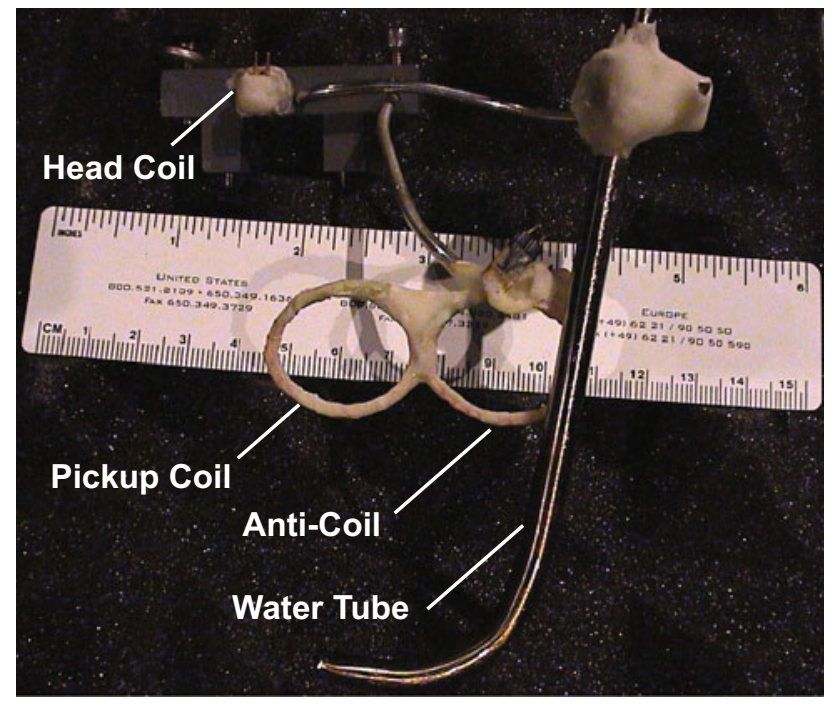

Fig. 1 Frontal view of the DMI assembly. Pickup coil, anti-coil and head coil are embedded in dental cement for protection. The water tube used to deliver the liquid reward, the pickup coil and anti-coil are fixed via two small steel wires to a plastics bridge that can be screwed into threaded small cylinders implanted on the monkey's skull. The head coil is glued to this bridge and is also embedded in dental cement. Note that in this picture the small laser pointer is not shown; its mounting point is above the head coil

magnetic fields produced by the ring. However, the primary fields also directly generate a much stronger induction voltage in the pickup coil, which for the horizontal field is given by

$V_{\text {PRIM, } \mathrm{H}}(\gamma, t)=L_{\text {coil }, \mathrm{H}} \cdot \sin \left(\omega_{\mathrm{H}} t\right) \cdot \sin (\gamma)$

with $L_{\text {coil, } \mathrm{H}}$ the coil's self-induction, and $\gamma$ the horizontal head orientation (similar for the vertical field, and for vertical head orientations, $\delta$, and for the frontal field, with $\cos \left(\sqrt{\gamma^{2}+\delta^{2}}\right)$ as flux $)$. In the DMI method, these primary field contributions are cancelled by a second coil (the anti-coil), connected in antiphase and ideally placed exactly co-planar with the pickup coil, and with exactly the same self induction (see Fig. 1). In that case, the total net voltages of the entire system will follow Eq. 10.

However, in practice, the magnetic fields are (i) neither perfectly homogeneous, (ii) nor perfectly orthogonal, (iii) the coils are not exactly co-planar, (iv) their self-inductions will not be identical, and (v) the centres and planes of pickup coil and ring will not be exactly aligned. The whole thing results in two important deviations from the ideal Eq. 10.

First, a net head-orientation-dependent voltage from the primary fields remains that may be quite complex, but is here approximated by

$$
\begin{aligned}
V_{\mathrm{NET}, \mathrm{H}}(\gamma, t)= & L_{\mathrm{coil}, \mathrm{H}} \cdot \sin \left(\omega_{\mathrm{H}} t\right) \cdot[\sin (\gamma)-\sin (\gamma+\Delta \phi)] \\
V_{\mathrm{NET}, \mathrm{V}}(\delta, t)= & L_{\mathrm{coil}, \mathrm{V}} \cdot \sin \left(\omega_{\mathrm{V}} t\right) \cdot[\sin (\delta)-\sin (\delta+\Delta \theta)] \\
V_{\mathrm{NET}, \mathrm{F}}(\gamma, \delta, t)= & L_{\mathrm{coil}, \mathrm{F}} \cdot \sin \left(\omega_{\mathrm{F}} t\right) \cdot\left[\cos \left(\sqrt{\gamma^{2}+\delta^{2}}\right)\right. \\
& \left.-\cos \left(\sqrt{\gamma^{2}+\delta^{2}}+\Delta \psi\right)\right]
\end{aligned}
$$

in which $L_{\text {coil, (H,V,F) }}$ is the coil's self induction for the three fields, and the misalignment of the coils is parametrised by angular differences $\Delta \Phi, \Delta \theta$, and $\Delta \psi$, respectively.

Second, the ring-coil misalignment is more serious, as it directly affects the DMI-shape factor, $L[\cos (x)]$ (which was derived for the ideal aligned case). In that case, the Biot-Savart law is no longer helpful, as the shape factor changes for each particular misalignment in 3D space, which in practice cannot be determined exactly. The coil-ring misalignment introduces a shift in the monotonic measurement range (e.g. from $[-30,+30]^{\circ}$ for the aligned situation, to $[-10,+50]^{\circ}$ for a particular misaligned situation). To simplify the math for our signal simulations we approximated this shift by introducing a phase angle, $\psi$, in the shape factor:

$L[\cos (\alpha)] \Rightarrow L[\cos (\alpha+\psi)]$

Our measurements (Figs. 8,9) show that the set of signal simulations (Eq. 1) based on these heuristic approximations (Figs. 2, 3) are a reasonable approximation of the actual situation.

\subsection{Simulation of head-unrestrained double magnetic induction signals}

The simulated gaze shifts and head saccade signals of Eq. 3 were used to calculate the resulting time-averaged (dc) DMI signals from the horizontal, vertical and frontal fields, as given by the analysis above, Eqs. 10-13:

$$
\begin{aligned}
V_{\mathrm{H}}(\alpha, \gamma, \varepsilon, \delta)= & K_{\mathrm{H}} \cdot \sin (\alpha+\gamma) \cdot L\left[\cos \left(\alpha+\psi_{\mathrm{H}}\right)\right] \\
& +V_{\mathrm{NET}, \mathrm{H}}(\gamma) \\
V_{\mathrm{V}}(\alpha, \gamma, \varepsilon, \delta)= & K_{\mathrm{V}} \cdot \sin (\varepsilon+\delta) \cdot L\left[\cos \left(\varepsilon+\psi_{\mathrm{V}}\right)\right] \\
& +V_{\mathrm{NET}, \mathrm{V}}(\delta) \\
V_{\mathrm{F}}(\alpha, \gamma, \varepsilon, \delta)= & K_{\mathrm{F}} \cdot \cos \left(\sqrt{(\alpha+\gamma)^{2}+(\varepsilon+\delta)^{2}}\right) \\
& \cdot L\left[\cos \left(\alpha+\psi_{\mathrm{H}}\right)\right] \cdot L\left[\cos \left(\varepsilon+\psi_{\mathrm{V}}\right)\right] \\
& +V_{\mathrm{NET}, \mathrm{F}}(\gamma, \delta)
\end{aligned}
$$

for the horizontal $\left(V_{\mathrm{H}}\right)$, vertical $\left(V_{\mathrm{V}}\right)$ and frontal $\left(V_{\mathrm{F}}\right)$ field signals, respectively. To simplify the implementation we assumed the same time-averaged constant $K$ for all three fields, which essentially means that the amplifiers were tuned such that they generated the same signal strengths. To further simplify the equations we took the geometric offset angles of Eq. 13 the same for the horizontal and vertical fields. We also 

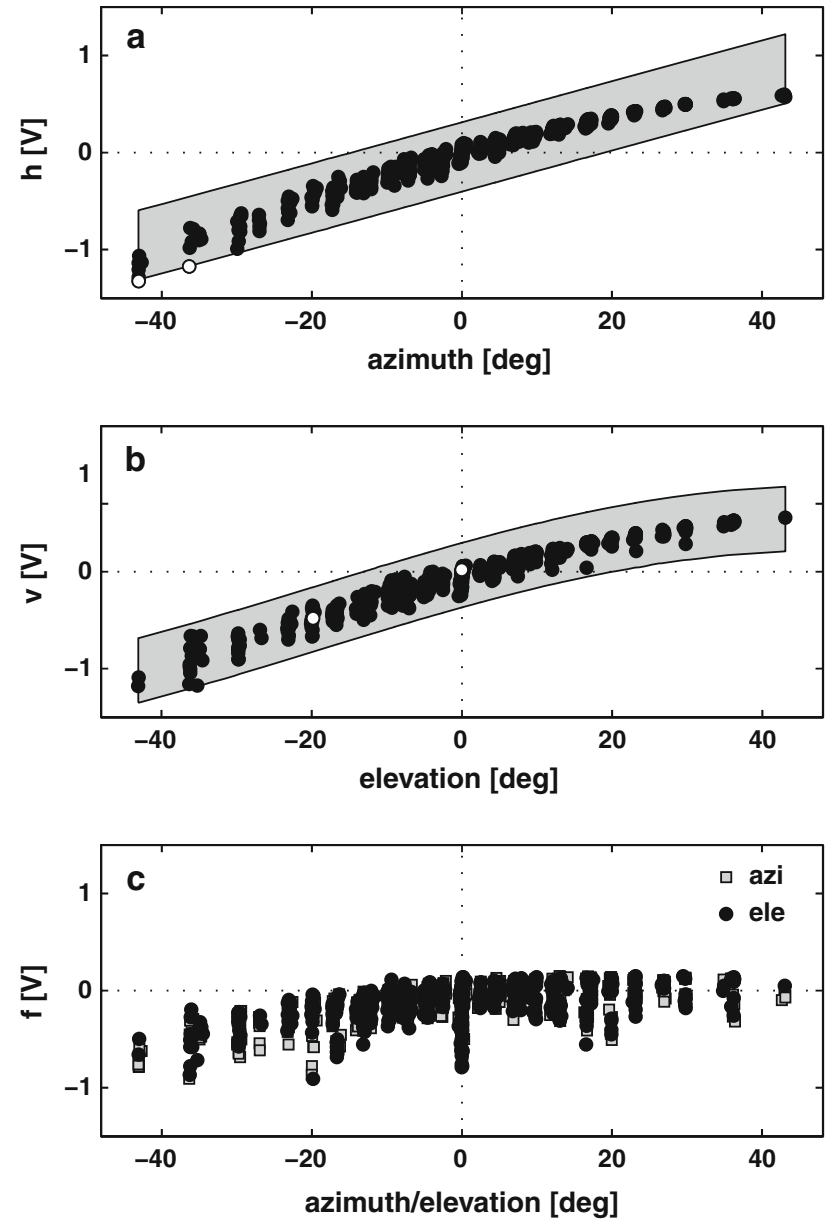

Fig. 2 Simulated DMI endpoint signals for the horizontal (a), vertical (b) and frontal (c) field components. a Horizontal voltages of simulated endpoints as a function of azimuth. The black circles indicate data points. A sine was fit to the data, to remove outliers. The grey patch depicts the area of one SD around the fit. Open circles indicate data points that fell outside this selection criterion. Note that in the simulations, all endpoints were correct by definition. The employed criterion can thus be considered conservative. b the same as in (a) but for the vertical component. c Frontal voltages as a function of either azimuth (grey squares) or elevation (black circles). For a detailed description, see text

took the self-inductions and phase shifts $\Delta \Phi, \Delta \theta$ and $\Delta \psi$ of Eq. 12 identical. Parameter values are given in Table 2.

\subsection{Target generation for the calibration of simulated gaze shifts}

In the actual experiments, we made use of a LED board attached to the wall of the experimental chamber (see Sect. 2.6.3). To enable a quantitative comparison between simulations and experimental results, we simulated gaze shifts towards targets on this LED board. Target locations were defined in a polar coordinate system. Eccentricity, $R$, is measured as the angle with respect to $0^{\circ}$ (straight ahead), whereas direction, $\Phi$, is measured relative to the horizontal meridian. Targets could be at $R=0,5,9,14,20,27,35$, $43^{\circ}$ (rings) and $\Phi=0,30,60, \ldots, 330^{\circ}$ (spokes). To present LED targets at intermediate locations, we used a rotating hoop with 32 LEDs $\left(5^{\circ}\right.$ spacing in elevation: -70 to $85^{\circ}$ ) and an azimuth rotation resolution of $<0.1^{\circ}$ (range -90 to $90^{\circ}$ ). For simulations and easy visualisation, we transformed polar coordinates $(R, \Phi)$ into the corresponding azimuth $(\alpha)$ and elevation $(\varepsilon)$ angles (Hofman and Van Opstal 1998) by

$\alpha=\arcsin (\sin R \cdot \cos \phi) \quad$ and $\quad \varepsilon=\arcsin (\sin R \cdot \sin \phi)$

respectively. Targets were randomly drawn from these locations with the restriction that the target location did not exceed the field-of-view $\left(<50^{\circ}\right)$ relative to the current fixation LED. The fixation location of the first trial was chosen randomly within the field-of-view from straight ahead. For all subsequent trials, the target location of the previous trial was taken as the new fixation location, i.e. endpoint and starting point of subsequent saccades were connected. This scheme yielded a combination of saccades with different start- and end positions and different amplitudes and directions that sampled the whole frontal hemisphere between $\pm 43^{\circ}$.

\subsection{Calibration of double-magnetic induction data}

A successful calibration of the DMI signals requires endpoints corresponding to known target locations. We aimed to extract the maximum amount of unique eye-head combinations from any given trial. To achieve this, we used the fact that the head typically lags the eye: i.e. the head is still moving when gaze has already reached the target. We selected all simulated samples after the head had reached its peak velocity. In the simulated data set, this amounted to about 183,000 unique eye-head combinations from a total of 400,000 samples (45\% [see also Sect. 2.1.1]).

We calibrated the selected gaze endpoints by applying two three-layer feed forward neural networks, one for the azimuth component and one for the elevation component. The five input units of the network were (1) $V_{\mathrm{H}}$, (2) $V_{\mathrm{V}}$, (3) $V_{\mathrm{F}}$, (4) simulated $\gamma$ and (5) simulated $\delta$, while the desired, single output of the network was the gaze angle, $\alpha$ or $\varepsilon$ that corresponded to the target coordinates. The networks were trained using the Bayesian-Regularization implementation of the back-propagation algorithm (Matlab 7.0, Neural Networks Toolbox, The Mathworks, Inc.) to avoid over-fitting (MacKay 1992). The trained networks were used to calibrate the remaining gaze shift samples, here called the test set $(55 \%$ of the data set). To assess the performance of the networks, the calibrated test set was compared to the simulated gaze signal by calculating the error as the difference between simulated and calibrated sample. The mean error and the 
Fig. 3 Simulated DMI endpoints before (a) and after (b) calibration. a $2 \mathrm{D}$ plot of the simulated raw horizontal and vertical voltages. Note the pronounced signal asymmetry. b After calibration, the pronounced signal asymmetry has disappeared. The endpoints can be mapped to the target locations accurately

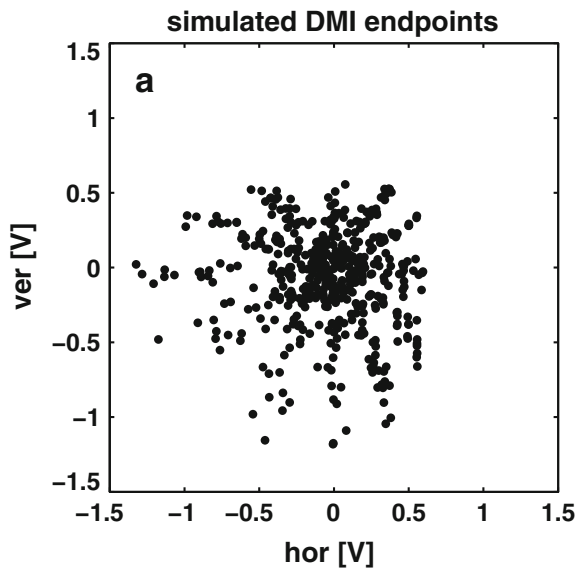

Table 2 Parameters for the simulation of the double-magnetic induction signals

\begin{tabular}{|c|c|c|}
\hline Parameter & Value & Description \\
\hline$N_{\text {coil }}$ & 100 & Number of turns of the pickup coil \\
\hline$N_{\text {ring }}$ & 1 & Number of turns of the ring \\
\hline$\omega$ & $75 \times 10^{3} \times 2 \times \mathrm{pi}$ & Angular frequency of the driving primary magnetic field in rad \\
\hline$B$ & $10^{-4}$ & Magnetic field strength in $\mathrm{T}$ \\
\hline$\mu_{0}$ & $1.26 \times 10^{-6}$ & Vacuum permeability/magnetic constant in $\mathrm{H} / \mathrm{m}$ \\
\hline$R_{\text {coil }}$ & $2.5 \times 10^{-2}$ & Radius of the pickup coil in $\mathrm{m}$ \\
\hline$R_{\text {ring }}$ & $0.8 \times 10^{-2}$ & Radius of the ring in $\mathrm{m}$ \\
\hline$R_{\text {eye }}$ & $1.2 \times 10^{-2}$ & Radius of the eye in $\mathrm{m}$ \\
\hline$Z_{\text {elec }}$ & $1.26 \times 10^{-3}$ & Electric impedance of the ring in $\Omega$ \\
\hline$d$ & $2 \times 10^{-2}$ & Coaxial distance between ring and pickup coil in $\mathrm{m}$ \\
\hline$L_{\mathrm{cl}}$ & 2.5 & Constant that is proportional to the coils' self-induction in a.u. \\
\hline$\Delta \Phi$ & 250 & $\begin{array}{l}\text { Phase shift representing the angle between the pickup coil } \\
\text { and anti-coil for the horizontal, vertical and frontal fields } \\
\text { in deg }\end{array}$ \\
\hline$\psi$ & 2 & $\begin{array}{l}\text { Misalignment between centres of rotation of the ring and } \\
\text { pickup coil for the horizontal, vertical and frontal fields } \\
\text { in deg }\end{array}$ \\
\hline
\end{tabular}

mean SD over all the samples and trials were used to assess the influence of (1) the number of units in the hidden layer, (2) the number of trials, i.e. the number of unique eye-head combination samples and (3) the reproducibility of calibration (see Fig. 4).

\subsection{Experiments}

\subsubsection{Subjects}

Behavioral experiments were conducted with three adult male rhesus monkeys (Macaca mulatta; MA, WO and OR, weight 7-9 kg, respectively). All experiments were conducted in accordance with the European Communities Council Directive of November 24, 1986 (86/609/EEC) and were approved by the local ethics committee (dier experimenten commissie, DEC) of the Radboud University Nijmegen for the use of laboratory animals. In each recording session, the monkey earned small water rewards per successful trial until it was satiated. Shortly before $(\sim 24 \mathrm{~h})$ the start of recording sessions, daily water intake was limited to approximately $20 \mathrm{ml} / \mathrm{kg}$, which follows the water-restriction protocol of the University of California at Davis, CA (AUCAAC 2001).

\subsubsection{Eye-head recording apparatus}

The monkey was seated in a completely dark room (approximately $2.5 \mathrm{~m} \times 2.5 \mathrm{~m} \times 2.5 \mathrm{~m}$ ) facing a light-emitting diode (LED) board used for stimulus presentation. Three orthogonal pairs of single-turn field coils (cross section of the wires six $\mathrm{mm}^{2}$ ) generated the primary magnetic fields and were mounted alongside the edges of the four walls, ceiling and 

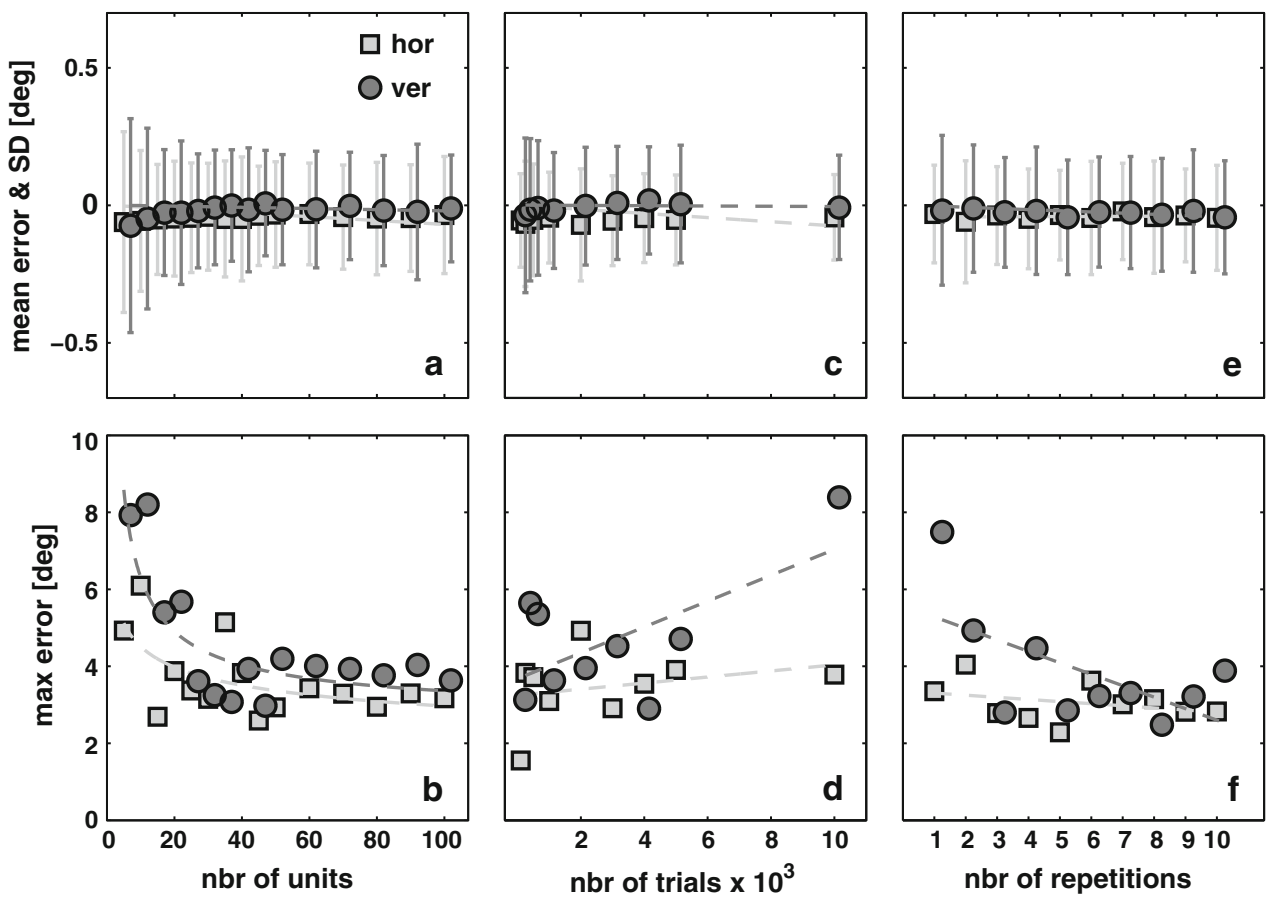

Fig. 4 Best parameters for use with the artificial neuronal networks obtained with simulated gaze shifts. Data corresponding to the horizontal network are denoted with light grey squares and those that belong to the vertical network as dark grey circles. Note that the vertical data are shifted to increase visibility. a Mean error, i.e. simulation-calibration, and SD of the error as a function of network number of hidden units. b Maximal error, i.e. simulation-calibration, as a function of network number of hidden units. $\mathbf{c}$ Mean error and SD of the error as a function

floor in a Helmholtz configuration. The coils were driven by custom-built amplifiers (horizontal field: $48 \mathrm{kHz}$, vertical field: $60 \mathrm{kHz}$, frontal field: $80 \mathrm{kHz}$ ). To monitor the monkey's eye movements, a golden ring that fitted around the cornea without obstructing the extraocular muscles was placed onto the sclera underneath the conjunctiva in an aseptic surgery (Judge et al. 1979; Bour et al. 1984). In order to measure the induction voltages from the secondary magnetic fields from the ring, a DMI assembly (Bremen et al. 2007b) was attached to a head holder that had been implanted onto the skull in an earlier surgery (Fig. 1). The assembly consisted of a pickup coil connected antiparallel to the anti-coil (both 100 turns, wire diameter $0.1 \mathrm{~mm}$, inner diameter $2.5 \mathrm{~cm}, R=37 \Omega$, $L=983 \mu \mathrm{H}$ at $1 \mathrm{kHz}$ ). The two coils, shaped roughly as an ellipse, were cemented into a figure-eight shape so that both laid in exactly the same plane. The pickup coil was positioned in front of the implanted eye while the anti-coil protruded temporally (like a monocle). Note that in this way the other eye was completely unobstructed, which differs from the DMI assembly that we have used previously on human subjects (Bremen et al. 2007b). In this way, a second pair of coils can be mounted in front of the other eye to measure of number of trials, i.e. endpoints, used for calibration (500 trials, 40 hidden units per network). d Maximal error as a function of number of trials, i.e. endpoints, used for calibration ( 500 trials, 40 hidden units per network). e Mean error and SD of the error as a function of number of repetitions (500 trials, 40 hidden units per network). f Maximal error as a function of number of repetitions (500 trials, 40 hidden units per network)

binocular eye movements. The distance between eye and pickup coil was approximately $1 \mathrm{~cm}$. To monitor head movements, we attached an additional small search coil to the DMI assembly. Finally, we mounted a small laser pointer (LQB-1-650, World Star Tech, Toronto, ON, Canada) to the assembly. This laser could be used to align the monkey's head with the LEDs: a procedure that we employed to calibrate the head coil (see Sect. 2.6.4.2). For liquid reward delivery, a thin stainless steel tube was mounted on the DMI assembly to which a silicone rubber tube could be attached once the primate chair was in place. The tube was connected to a water reservoir and a latch outside the experimental chamber. All wires and the water tubing were led from the top of the monkey's head to a custom-built tether system consisting of a metal rod attached to the monkey chair and a plastic ring (diameter $5 \mathrm{~cm}$ ) through which the cables were led. The whole assembly without the tether system weighed about 50 grams.

The outputs of the DMI assembly and the head coil were connected to six lock-in amplifiers (Princeton Applied Research, Model 128A). Three were used for the horizontal, vertical and frontal field signals of the DMI assembly 
and the other three for the head coil. These signals were low-pass filtered at $150 \mathrm{~Hz}$ (4th order Butterworth, custombuilt), digitised at a rate of 1017.25 samples/s (RX6 System 3, Tucker Davis Technologies; Alachua, Florida, USA) and finally stored on a computer's hard disk for subsequent offline analysis.

\subsubsection{Stimuli}

The monkey faced a frame (radius: $149 \mathrm{~cm}$ ) with twelve spokes and seven concentric rings (LED board) mounted on the wall of the experimental chamber. LEDs $\left(0.2^{\circ}\right.$ diameter as viewed by the subject, intensity $0.5 \mathrm{~cd} \mathrm{~m}^{-2}, \lambda=565 \mathrm{~nm}$ ) were mounted at $R=0,5,9,14,20,27,35,43^{\circ}$ (rings) and $\phi=0,30,60, \ldots, 330^{\circ}$ (spokes) on the frame (see Sect. 2.1.3).

Target selection and data acquisition were done with an in-house program written in $\mathrm{C}++$ and Matlab running on a PC $(2.8 \mathrm{GHz}$ Intel Pentium D, Dell, Windows XP). To assure microsecond timing precision, trial information was sent from the PC to a micro-controller that worked as a latch initiating a trial when all hardware components were ready.

\subsubsection{Paradigms}

All experiments were conducted in darkness. One recording session lasted for several hours, depending on the monkey's motivational level. At the beginning and at the end of an experimental session, the monkey's head was fixed to the primate chair via a custom-built stainless steel rod that could be attached to the head holder on the monkey's skull. After head fixation, the DMI assembly - with head coil, laser pointer and reward system — was mounted/removed. Since all components were attached to skull-fixed anchor points, the rigid DMI assembly could be mounted in a reproducible way between consecutive recording sessions.

2.6.4.1 DMI calibration paradigm We trained monkeys on a simple visual fixation task by instrumental conditioning. To initiate a trial the head-unrestrained monkey had to press a handle bar. A randomly selected LED was lit, extinguished after $600-1,100 \mathrm{~ms}$, upon which a different LED was illuminated. This sequence was repeated for a random number of LEDs (up to six). The last LED in a sequence changed its intensity after a randomly selected duration. The monkey had to respond to this intensity change as quickly as possible by releasing the handle bar within $600 \mathrm{~ms}$. All LEDs in the trial were lit for a randomised period of time (between 600 and $1,100 \mathrm{~ms})$. The trial was aborted when the monkey released the bar too early, i.e. before the intensity change.

In case of a correct trial, the monkey was rewarded with approx. $0.2-0.4 \mathrm{ml}$ of fresh water. We increased (decreased) task difficulty by decreasing (increasing) the LED intensity change. To perform well, the monkey needed to closely follow and foveate the LEDs. In addition, we monitored the monkey's behaviour via an infrared video camera, bar-release reaction times, and overall performance. This was especially important in the initial stages of training when we could not yet rely on calibrated eye and head signals.

Note that the definition of a trial differs for simulations and measurements. While in the simulations one trial contains one target and saccade, in the measurements, one trial could contain up to six targets and saccades. On a typical recording day, the monkey would make about 1,500-2,000 gaze shifts.

2.6.4.2 Head-coil calibration paradigm It is important to note that the calibration of the DMI signal (gaze in space) can be done with uncalibrated head-coil signals. However, to determine eye-in-head orientations from the calibrated gaze traces (e.g. to analyse the vestibular-ocular reflex during gaze shifts) the head coil needs to be calibrated too. Since the headcoil signals have a simple sinusoidal relationship with head orientation (e.g., Eq. 11), this procedure is relatively straightforward. We manually aligned the monkey's head with the head-fixed laser pointer to the LEDs on the board. Owing to physical constrains, it was not possible to align the head with the most eccentric LED ring $\left(R=43^{\circ}\right)$ and with the upward $R=35^{\circ}, \phi=90^{\circ}$ LED. We sampled $500 \mathrm{~ms}$ of fixation for a given LED, and the monkey was rewarded after each LED with approx. 0.2-0.4 $\mathrm{ml}$ water via the head-fixed drinking tube. The monkey rapidly adapted to the procedure and after having fixated a few LEDs in this way, he actively cooperated with the experimenter. Collection of one calibration set took about 3-5 min.

\subsubsection{Calibration}

Combinations of raw head-movement data (AD values from the horizontal and vertical channels) and known LED locations (azimuth and elevation, in deg) were used to train two three-layer neuronal networks for head-azimuth and elevation, respectively. The networks comprised two input units $\left(V_{\mathrm{H}}-V_{\mathrm{F}}\right.$ or $\left.V_{\mathrm{V}}-V_{\mathrm{F}}\right)$ four hidden units, and one output unit $(\gamma$, or $\delta)$ and were trained in the same way as the gaze networks. In addition to a nearly linear mapping from $\mathrm{AD}$ values to degrees, the networks also accounted for small inhomogeneities in the fields and cross-talk between the channels. The trained networks were used to calibrate the experimental head data yielding the $\gamma$ and $\delta$ angles.

The recorded ring signals were calibrated employing the same network architecture as described for the simulated data. Before calibration, data were digitally low-pass filtered at $75 \mathrm{~Hz}$ using a 50-point FIR (finite impulse response) filter. The networks were trained on a subset of the sampled data. Gaze-shift and head-saccade endpoints were extracted 
as described in Sect. 2.6.3. However, the velocity criteria were applied to the raw ring and head coil data. Note that raw head-saccade endpoints were used in the input layer.

\subsubsection{Data analysis}

We calculated the spatial resolution of the DMI method by determining the SD over the fixation epoch for each target location individually and converted the obtained values to minutes of arc. In order to resolve a movement, the signal must be larger than the SD. Note that the recorded and calibrated signals do contain not only system noise but also minute fixational movements of the eye.

Unless stated otherwise, all fits employed the least-squares criterion.

\section{Results}

\subsection{Simulations}

\subsubsection{Raw DMI signals}

Figure 2 depicts simulated DMI signals of the horizontal (Fig. 2a), vertical (Fig. 2b) and frontal (Fig. 2c) field as a function of gaze azimuth and elevation angle $(N=492)$. The simulations accounted for a $2^{\circ}$ (see Table 1 ) misalignment of ring and pickup coil as well as for a head-orientation-dependent $V_{\text {NET }}$ function (see Methods, 2.1.1, and Table 1). The pronounced nonlinearity of the signals is particularly obvious for the horizontal and vertical fields. While the linear range is shifted to negative azimuth and elevation angles, the nonlinear range starts already around $0^{\circ}$. The strong reduction in measurement range for rightward gaze positions is caused by the misalignment between the centres of ring and pickup coil. The frontal field signals are plotted as a function of azimuth (grey squares) and of elevation (black circles). Note that the emerging pattern is more complex. Owing to the influence of the $V_{\mathrm{NET}}$ signal, data points are tilted around $0^{\circ}$ azimuth/elevation. For a comparison with measured data compare Fig. 2 to Fig. 8.

In order to check for endpoints that were extracted incorrectly, we fitted a cosine through the data and discarded all data points that fell outside $\pm 1 \mathrm{SD}$. The grey patches in Fig. $2 \mathrm{a}$ and $\mathrm{b}$ indicate this range. Two data points were discarded (plotted as open circles) as they failed to meet the criterion (Fig. 2a). This procedure was designed to minimise the inclusion of wrong endpoints for the training of the artificial neuronal networks. As can be seen, this approach is conservative since in the simulations all endpoints were extracted correctly.

In Fig. 3a, we have plotted uncalibrated endpoints of the vertical field versus those of the horizontal field. The pronounced nonlinearity and asymmetry in the data can be clearly seen as the data points for positive horizontal and vertical voltages are squeezed while the negative range is expanded. A similar pattern can be observed for the measured data (Fig. 9a).

\subsubsection{Calibrated gaze signals}

Figure $3 \mathrm{~b}$ shows the gaze endpoints of Fig. 3a after calibration with two three-layer artificial neuronal networks, each with 40 hidden units. Target locations are indicated by grey squares and calibrated endpoints as black circles connected with black lines to their respective target. The horizontal and vertical networks were well able to map the complex, nonmonotonic DMI signals. The mean error, i.e. target location minus calibrated endpoint, for the horizontal component over 500 trials was $0.00^{\circ}$ with a SD of $0.17^{\circ}$ and for the vertical component $0.00^{\circ}$ with a SD of $0.13^{\circ}$.

In order to determine the best settings for the artificial neuronal networks, we performed several optimisation simulations on the entire data set. First, we determined the optimal number of hidden units by running the calibration routine with different numbers of hidden units (5-50 in steps of 5, $60-100$ in steps of 10) for 1,000 simulated trials. This procedure was repeated three times, and the resulting mean error and the SD are shown in Fig. 4a. The maximal error is shown in Fig. 4d. The data of the vertical component (dark grey circles) were shifted to the right with respect to the horizontal data (light grey squares) for graphical purposes only. The mean error was smaller than $0.05^{\circ}$ for both horizontal and vertical components and did not vary with the number of units. In fact, linear fits for both components (dashed grey lines, vertical fit occludes horizontal fit) had a slope and an offset of $0^{\circ} /$ unit and $0^{\circ}$, respectively. The SD of the error, however, decreased exponentially with the number of units and reached its minimum at about $30-40$ units. We therefore chose 40 units for all subsequent networks.

Second, an important aspect of the simulations was to assess the minimum number of trials needed for a good calibration of the DMI signals. We therefore simulated a varying number of gaze shifts and calibrated the extracted endpoints. To assess the influence of the number of training trials on performance-and therefore the number of unique eyehead combinations-we simulated and calibrated experiments with 100,500,1,000-5,000 (steps of 1,000) and 10,000 trials. Figure $4 \mathrm{~b}$ and e show the mean error, SD of the error and maximal error as a function of number of gaze shifts. Mean error (close to $0^{\circ}$ ) and SD $\left(<.25^{\circ}\right)$ did not vary with the number of trials. Note that the number of trials did not affect the mean error either. The SD decreased slightly and reached its minimum at about 3,000 trials. Further increasing the number of trials did not improve performance. The maximum error oscillated between 1.5 and $6^{\circ}$. The data point for 

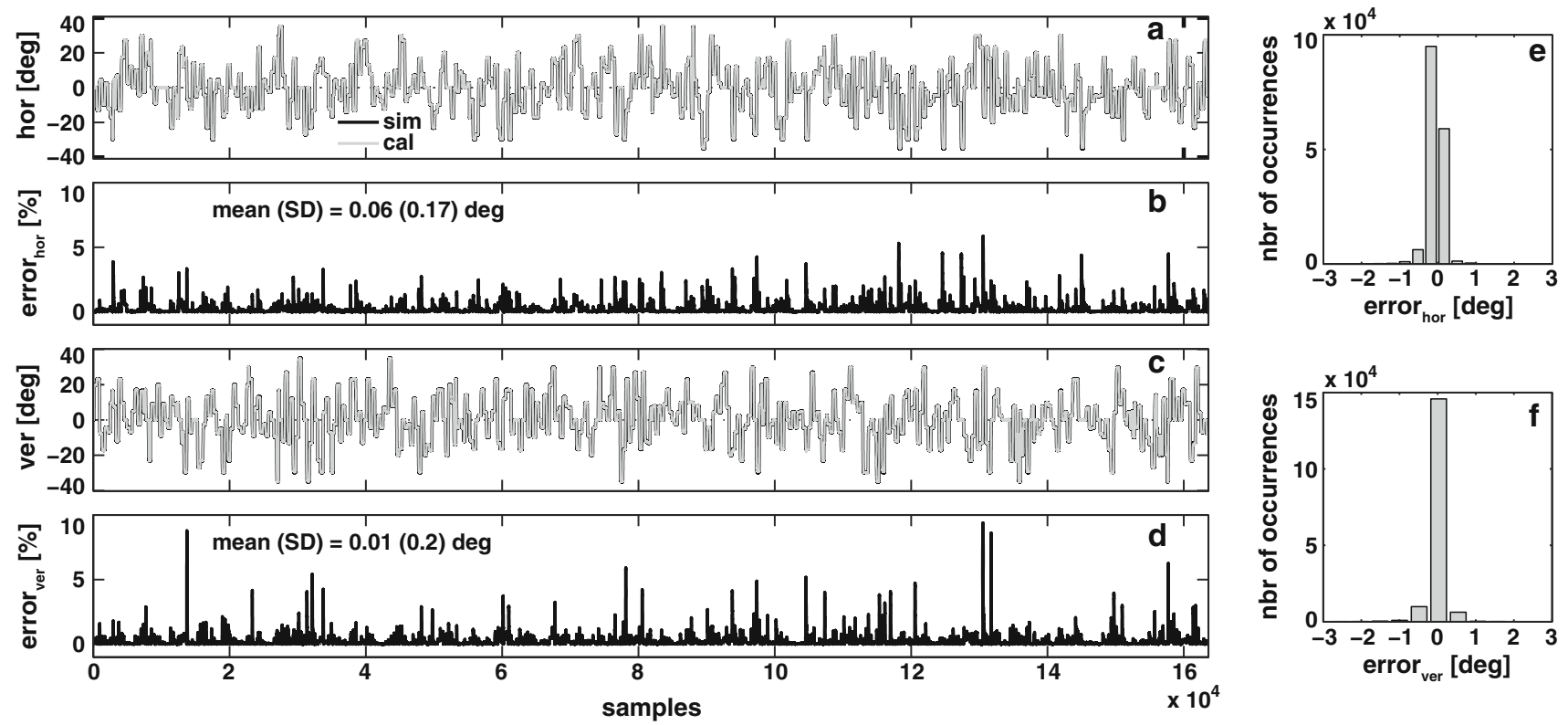

Fig. 5 Overview of all simulated (black line) and calibrated (thin grey line) saccades in one continues trace for the horizontal (a) and vertical (c) components. Relative errors, i.e. simulation-calibration in percent, are shown in (b) for the horizontal and in (d) for the vertical components.
The error for both components is always smaller than $10 \%$. Histograms of the error in degrees are depicted in (e) and (f) for the horizontal and vertical components, respectively. The majority of errors are in the order of $\pm 1^{\circ}$ the vertical network at 10,000 trials is due to an outlier in one of the three repetitions. In the other two runs the maximum error was close to $4 \mathrm{deg}$. This demonstrates a typical issue of artificial neuronal networks. The network could get trapped in a local minimum that results in a badly trained network. For the simulations, we chose 500 trials as a cut-off training length to limit computational time.

Third, to test the robustness/reproducibility of the networks, we repeated the network training for 500 simulated trials with 40 hidden units 10 times. As can be seen in Fig. 4c, the mean error and SD did not vary appreciably with the number of repetitions. However, the SD between repetitions did vary to some degree (compare for example repetition 1 with 3 of the vertical component). Clearly, large SDs correspond to larger maximum errors and smaller SDs to smaller maximum errors (Fig. 4f). It is, therefore, advisable to select networks in the training process with a small SD of the error for both components.

So far, we have only discussed calibrated endpoints obtained after training the networks. Figure 5a and d depicts 500 simulated (thick black line) and calibrated (grey lines) gaze shifts. As can be seen, the calibrated gaze shifts nicely follow the simulated horizontal (Fig. 5a) and vertical components (Fig. 5c). A more quantitative analysis is provided by Fig. $5 b$ and d, which show the sample-to-sample difference between simulation and calibration, in percent for the horizontal (Fig. 5b) and the vertical component (Fig. 5d). The error never exceeded $10 \%$, but note that large errors were very rare, as can be better appreciated from the histograms of Fig. 5e (horizontal) and $\mathrm{f}$ (vertical). Note that in these subplots the error is given in degrees. The far majority of errors were at, or close to, $0^{\circ}$. The number of occurrences of errors larger than $\pm 1^{\circ}$ are not visible on the scale of the histograms ( $10^{5}$ samples horizontal; $1.5 \times 10^{5}$ samples vertical $)$. Overall, this shows that the networks were very well able to calibrate not only the gaze-shift endpoints (for which they were trained) but also the intermediate samples of simulated head-unrestrained gaze shifts that had not been not used in the training set.

As a further test for the quality of the calibration, we extracted saccade parameters from the simulated gaze shifts (prior to feeding them to the DMI conversion, Eq. 1) and compared them to the calibrated gaze shifts. We extracted saccade on- and offset, amplitude and peak gaze velocity. Figure $6 \mathrm{a}$ depicts the peak velocity as a function of amplitude. The original simulated data points are shown as black circles; calibrated data points as grey circles. The peak velocity versus amplitude relationship was fit with an exponential (red: simulated; blue calibrated). Data and fits for simulated and calibrated gaze shifts are in good agreement. At amplitudes above $40^{\circ}$, the calibrated data tend to slightly underestimate the peak velocity, but this may be due to the relatively small number of large saccades in the training sample. Figure $6 \mathrm{~b}$ shows the error in peak velocity between simulated and calibrated data as a function of amplitude (black circles). The running average is plotted through the data as a grey line 

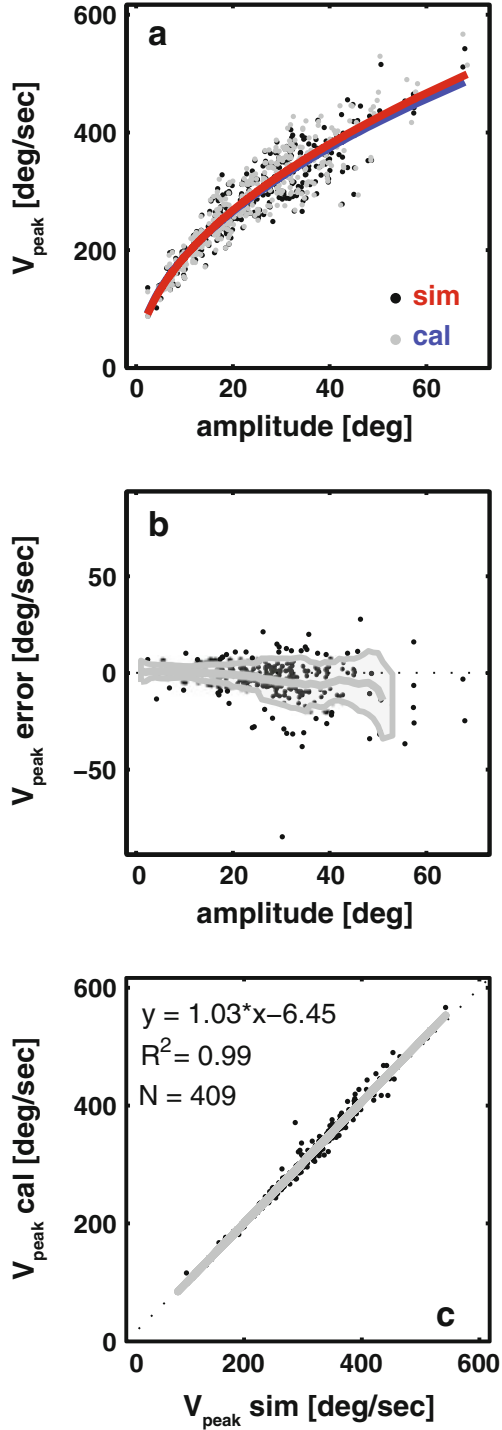

Fig. 6 Comparison of the main-sequence amplitude vs. peak-velocity relation for simulated (black circles) and calibrated (grey circles) gaze shifts. a Velocity plotted as a function of amplitude. Velocity increases exponentially with amplitude. Red line exponential fit to the simulated data and blue line fit to the calibrated data. $\mathbf{b}$ Difference between simulated and calibrated data as a function of amplitude. The grey line indicates the running average through the data and the patch covers 1 $\mathrm{SD}$ around the running average. $\mathbf{c}$ Linear regression analysis by plotting the calibrated peak velocity as a function of simulated peak velocity. Note the high correlation

and the patch indicates the SD of the error. Although the SD slightly increased with amplitude, the overall error remained very small (within about $10 \%$, which is the typical noise level). To further assess how well the calibrated data reflect the simulated gaze shifts we plotted peak velocity obtained from the calibration against peak velocity from the simulations in Fig. 6c. As can be seen the two highly correlate $\left(R^{2}=0.99\right)$. From these data we conclude that the calibration routine preserved the saccade kinematics well.

\subsection{Measurements}

Data presented in this section were collected from monkey MA on two successive recording days. Each session lasted for about $4 \mathrm{~h}$, during which the monkey completed a total of about 2,000 trials.

\subsubsection{Raw DMI signals}

Next we present measured gaze shifts and head saccades from monkey MA equipped with the DMI assembly shown in Fig. 1. Figure 7a shows the location and order of six targets presented to the monkey in a representative trial. Note that in the lower hemisphere, two rings of the setup at eccentricities $R=35$ and $43^{\circ}$ could not be used since the monkey was unable to see them. In this particular trial, the monkey had to follow six targets before the last target changed intensity and he had to release the bar for his reward. The whole trial lasted longer than $7 \mathrm{~s}$. The monkey's uncalibrated gaze shifts and head saccades are shown in Fig. 7b and c, respectively. The horizontal component is depicted as a black line, the vertical as a dark grey line and the frontal as a light grey line. The thick lines in different shades of grey at the bottom of each subplot indicate the on- and offset of each target. The shades correspond to Fig. 7a. Note that the traces both for the DMI method and the search coil (SC) are smooth and with little noise. Figure $7 \mathrm{~b}$ and $\mathrm{c}$ also illustrate how the endpoints for calibration were extracted. First, the offset of each target was determined; then, we applied a velocity criterion to the preceding 600 samples, i.e. gaze velocity had to be smaller than $300 \mathrm{mV} / \mathrm{s}$, a value determined after visual inspection of the uncalibrated velocity traces. Since neighbouring samples have similar values we selected every tenth sample from the original 600 samples to train the networks. The reason for not taking the mean over a given range of samples can be appreciated when looking at the head saccade made towards the third and fourth target. The head still moved while gaze was already on target, i.e. the eye-in-head counteracted the head movement through the operation of the VOR. These additional eye-head combinations are essential for a successful calibration (see Bremen et al. 2007b, and the simulation section, above). Extracted samples are indicated as thick lines in the shading corresponding to the respective field component.

Figure 8 depicts the extracted endpoints in the same format as Fig. 2. In Fig. 8a the horizontal DMI signal is plotted as a function of azimuth, the vertical component as a function of elevation in Fig. 8b, and the frontal component versus azimuth (grey squares) and elevation (black circles) in Fig. 8c. The endpoints were aligned such that the signal recorded at $0^{\circ}$ azimuth and elevation coincided with $0 \mathrm{~V}$. A comparison with Fig. 2 shows that the simulations nicely captured the main features of the recorded signals. The misalignment of 
Fig. 7 Uncalibrated measured DMI and head coil signals. a Stimulus array (open squares) and targets (circles in different shades of grey). The numbers close to the targets indicate the order of presentation.

b Recorded DMI position signals of the horizontal (black line), vertical (dark grey line) and frontal (light grey line) field. The thick grey bars at the bottom of the plot indicate the presentation of a target. The shades of grey correspond to the ones used in (a). c Same as (b) but for the head coil signal
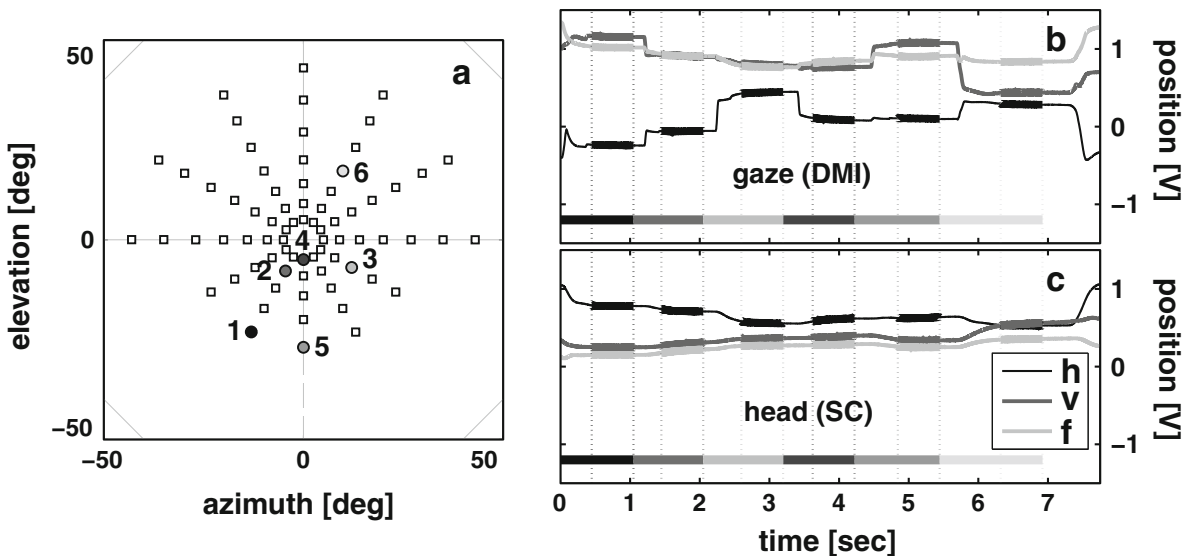
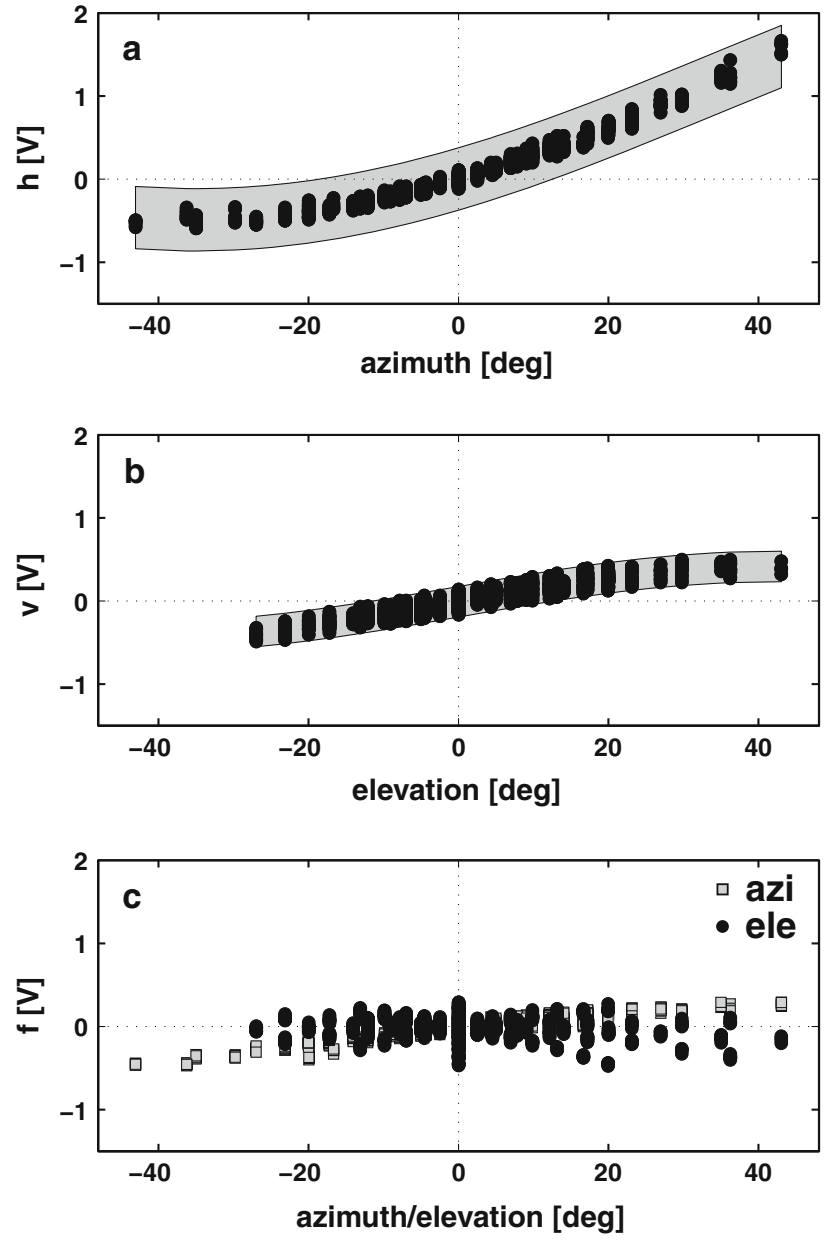

Fig. 8 Measured endpoints after extraction from the saccades. The convention is the same as used in Fig. 1

ring and pickup coil can be seen in Fig. 8a as a shift of the linear range towards positive azimuth angles while the signal became ambiguous for large negative azimuth locations. For the vertical component, some target locations are missing compared to the simulations since the monkey was unable to see these locations (see also Fig. 7a). The frontal field nicely exhibits the tilt due to the influence of $V_{\mathrm{NET}}$.

Fig. 9a plots the raw vertical gaze-shift component against the horizontal signal (note that the total DMI signal contained six channels). Again the pronounced non-monotonicity is seen for negative azimuth locations. The shape of the target LED board can be noted to some extent, but it is clear that the raw signals provided a highly distorted representation of the actual gaze shifts. Nevertheless, the neural networks could successfully map these rather complex signals onto the actual target array, as shown in Fig. 9b. Target locations are indicated by grey squares while the calibrated endpoints are shown as black circles. In total 82,655 of 3,311,057 (2.5\%) recorded samples of one session were used for this calibration. The mean error and SD for the horizontal component were $0.00^{\circ}$ and $0.87^{\circ}$, respectively. For the vertical component we obtained a mean error of $0.00^{\circ}$ and a SD of $1.99^{\circ}$.

\subsubsection{Calibrated DMI signals}

Fig 10 shows four complete examples of calibrated eye-head gaze shifts of monkey MA in the visual-jump paradigm (top halve of the plot), with the corresponding gaze- and head vectorial velocities in the lower halve of the figure. Note the clear correspondence of the 2D gaze-in-space and head-inspace movement trajectories. As expected, the head did not reach the target locations and typically lagged the gaze shifts (Goossens and Van Opstal 1997). Note that the velocity profiles (low-pass filtered at $80 \mathrm{~Hz}$ ) are smooth and with little noise. The gaze shifts (black thick line) were much faster (sometimes exceeding $800^{\circ} / \mathrm{s}$ ) than the head movements (red line; up to about $200^{\circ}$ /s) with larger gaze shifts exhibiting higher peak velocities.

An important aspect of the head-unrestrained DMI method for the use with laboratory animals is a reproducible fixation of the DMI assembly from session to session. To test whether 
this would indeed be the case for our monkey preparations, we first trained a network on data obtained from day A (set A) and used the resulting network to calibrate the data obtained on the subsequent day (set B). Figure 11 compares the raw data (left column) and calibrated data (right column) of set A versus set $\mathrm{B}$. The data were obtained by averaging endpoints of each set for each of the 74 LED locations.

Raw data endpoints for set A (black circles) and set B (dark grey diamonds) are shown in Fig. 11a. Note the close correspondence between the two sets collected on the two consecutive days. This can also be seen when looking at the individual fields. Figure $11 \mathrm{~b}$ plots the horizontal component of set $\mathrm{B}$ as a function of the horizontal component of set A. Figures 11c and d plots the data of the vertical and frontal field in the same manner. Data of all fields could be fit with a straight line. The coefficients of determination, $R^{2}$, for all three components were larger than 0.9 , with slopes being close to one and offsets close to zero.

In Fig. 11e the calibrated saccadic endpoints of set A (black circles) and set B (dark grey diamonds) are plotted in 2D together with the target location (light grey squares). Endpoints for the same target are connected with black lines. The two sets correspond well, with only very small differences. There were only three outliers with a difference exceeding $5^{\circ}$ (see also Fig. 11h). These outliers are likely attributable to incorrect target fixations by the monkey, because the endpoint of one set was close to the actual target location, while the endpoint of the other set was far offtarget.

A better comparison of the two sets is provided by Fig. 11f and g, which plot the azimuth (Fig. 11f) and elevation (Fig. 11g) components of set B against those of set A. Linear regression analysis on the azimuth components yielded a slope of 0.97 and an offset of $0.01^{\circ}$ with a $R^{2}$ of 1.00 . For the elevation component the values were 1.00 for the slope, $0.00^{\circ}$ for the offset and 0.99 for $R^{2}$.

The error, i.e. set A-set B, of the azimuth (black circles) and elevation (light grey circles) components is plotted as a function of location in Fig. 11h. The majority of errors was smaller than $\pm 5^{\circ}$. The mean error for both the azimuth and the elevation components were close to $0^{\circ}$ with SD's smaller than $2^{\circ}$. Taken together this demonstrates that the DMI assembly is fixed rigidly and reproducibly between successive recording sessions.

Figure 12 assesses the resolution (in minutes of arc) of the head-unrestrained DMI method for the horizontal (Fig. 12a) and vertical (Fig. 12b) movement components. The worst resolution was obtained in the upper left quadrant of the vertical component, and was about $30 \mathrm{~min}$ of arc. Typical values were smaller than $10 \mathrm{~min}$ of arc for both components (Fig. 12b,c). These values compare well to those previously reported for the DMI method and for the standard SSC technique (Bremen et al. 2007b).

\section{Discussion}

The data presented in this article demonstrate that the DMI method can be successfully applied to measure head-unrestrained gaze shifts in laboratory animals. Although we tested our method on trained rhesus macaques, there is no reason why the method could not be used in other experimental animals, like cats, squirrel monkeys, marmosets, or even smaller animals like ferrets, chinchillas or mice. The only requirement for the method to work is the ability of the animal to make instructed goal-directed gaze shifts toward visual target locations.

An important aspect of the current study is the introduction of a simple calibration routine that solely relies on accurate fixation of randomly presented visual targets in the range of interest. Before applying this calibration routine to real measurements obtained with monkeys, we performed realistic computer simulations that mimicked natural head-unrestrained gaze shifts with a physical model of the resulting DMI signals (Fig. 2). We showed that artificial neuronal networks could be efficiently trained to calibrate fixation endpoints with great accuracy (Fig. 3), but also that the networks were able to interpolate the remaining data points to precisely capture the whole saccadic gaze traces (Fig. 5), while even preserving the details of the main sequence relations, for which the most sensitive parameter is peak gaze velocity (Fig. 6).

The experimental data were calibrated successfully (Figs. 9, 10). In accordance with previous data (Bremen et al. 2007b) the worst spatial resolution was about $30 \mathrm{~min}$ of arc, which is well within the range of best precision of the monkey oculomotor system. The typical resolution of the gaze shifts, however, was below $20 \mathrm{~min}$ of arc and was therefore comparable to values that have been reported with the SSC technique (e.g. Bour et al. 1984).

\subsection{Training the neuronal networks}

The networks were trained on gaze endpoints and their inputs were the raw head orientation and the recorded voltages of the horizontal, vertical and frontal components induced by the ring. Note that it is not necessary to use calibrated head orientations as input to the networks. But in order to make full use of all recorded signals, including a reconstruction of eye-in-head orientation during gaze shifts, it is essential to calibrate the head coil as well. This is especially important for example in studies concerned with eye-head coordination, or with the study of the vestibulo-ocular reflex.

For a successful calibration of the data, it is important to feed endpoints into the networks with different gaze-head combinations. It is noteworthy that each subject has an idiosyncratic way of generating head-unrestrained gaze shifts, i.e. the contribution of the head to the total gaze shift varies 
Fig. 9 Measured DMI endpoints before (a) and after (b) calibration. a 2D plot of the simulated raw horizontal and vertical voltages. Note the pronounced signal asymmetry. b After calibration, the pronounced signal asymmetry disappears. The endpoints can be mapped to the target locations accurately. Compare with Fig. 2

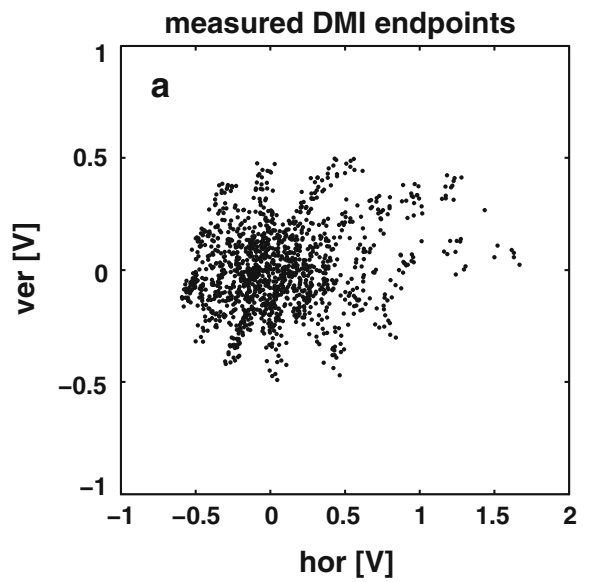

considerably from subject to subject (Fuller 1992); this was also observed in our monkeys (data not shown). The calibration routine deals with this idiosyncratic behavior in a natural way. Since the subject will produce the same combinations during calibration and experiment there is no need for a guided calibration paradigm, in which subjects have to make (unnatural) coordinated eye-head movements (Bremen et al. 2007b).

Note that the head coil data could in principle also be calibrated in a different way. The signals of the three magnetic fields (horizontal, vertical and frontal), and thus each head orientation $(\alpha$ and $\varepsilon$ ), fall on the surface of an ellipsoid. The axes of this ellipsoid are fully determined by the properties of the coils and the magnetic fields. The field strengths and amplifiers of the recording system could be tuned such that the data fall on the surface of a sphere. Calibration of the head coil data could then be achieved by fitting the offset-corrected data to the surface of a sphere. However, determining the optimal fit parameters for the surface of an ellipsoid or even a sphere is not straightforward (Gander et al. 1994; Turner et al. 1999) and crosstalk between the fields can lead to serious distortions of the data, which has to be accounted for as well. Although for the present study it was not necessary to implement this calibration scheme, it could be necessary for smaller laboratory animals, like mice, in which it is not so easy to align the head with the calibration target.

How the neural networks solve the mapping problem of DMI voltages into calibrated gaze positions has not been analysed in this article. Clearly, the inclusion of hidden units was essential, and each unit represents a mixture of the external inputs through its five input weights. In our first study on the DMI system, which dealt with the relatively simple, idealised one-dimensional version with only three inputs $\left(V_{\mathrm{H}}, V_{\mathrm{F}}\right.$ and $H_{\mathrm{H}}$ ), we noted that a network with three hidden units sufficed to solve the problem (Bremen et al. 2007a). For this case in question, it is relatively straightforward to understand how the network implements the required transformation, as each of the three hidden-unit inputs can be simply described by the inner product between the 3D input vector, $\vec{I}=\left[V_{\mathrm{H}}, V_{\mathrm{F}}, H_{\mathrm{H}}\right]$, and the weight vector, $\vec{W}=\left[w_{\mathrm{VH}}, w_{\mathrm{VF}}, w_{\mathrm{HH}}\right]$ (e.g. Krommenhoek et al. 1996), and thus yields a 3D hidden-unit output vector. In principle, such a 3D-3D mapping is unique, although the hidden-unit to output weighting (3D-1D) provides an additional degree of freedom, rendering the solution to depend on the initial conditions of the network. The situation gets more complicated when the number of independent inputs increases to five, like in the current 2D simulations, and the hidden units are trained to represent a highly nonlinear five-dimensional space. Our brute-force simulations of this simplified non-ideal geometry (Eq. 14) showed that the optimal number of hidden units fell between 20 and 40 (Fig. 4). Thus, the network's encoding of the 5D input space is far less trivial than for the idealised case. Because the number of hidden units exceeds the dimensionality of the input, there is no unique solution for this particular mapping problem (it is overdetermined). As each unit's input has a receptive field in a direction given by the inner product: $R_{k}=\sum_{n=1}^{5} w_{\mathrm{kn}} \cdot I_{n}$, and the final values of trained weights heavily depend on the initial weight values of the network, there are infinitely many ways in which the network can distribute the five inputs over the 20 or more hidden units. At the network's output unit, the total sum of hidden unit outputs monotonically relates to the desired gaze angle: $O=\sum_{\mathrm{k}=1}^{\mathrm{NHID}} w_{\mathrm{ok}} \cdot H_{\mathrm{k}} \propto G$. Again, the hidden-to-output weight values vary from simulation to simulation with the randomised initial conditions.

Because the problem is overdetermined, a full analysis of the properties and tunings of the hidden units is beyond the scope of this study, the primary aim of which was to provide a pragmatic black-box approach to solve the problem.

\subsection{Stability of the assembly}

Owing to anatomical differences around the nose and eyes, and to idiosyncratic differences of the head implants, the 

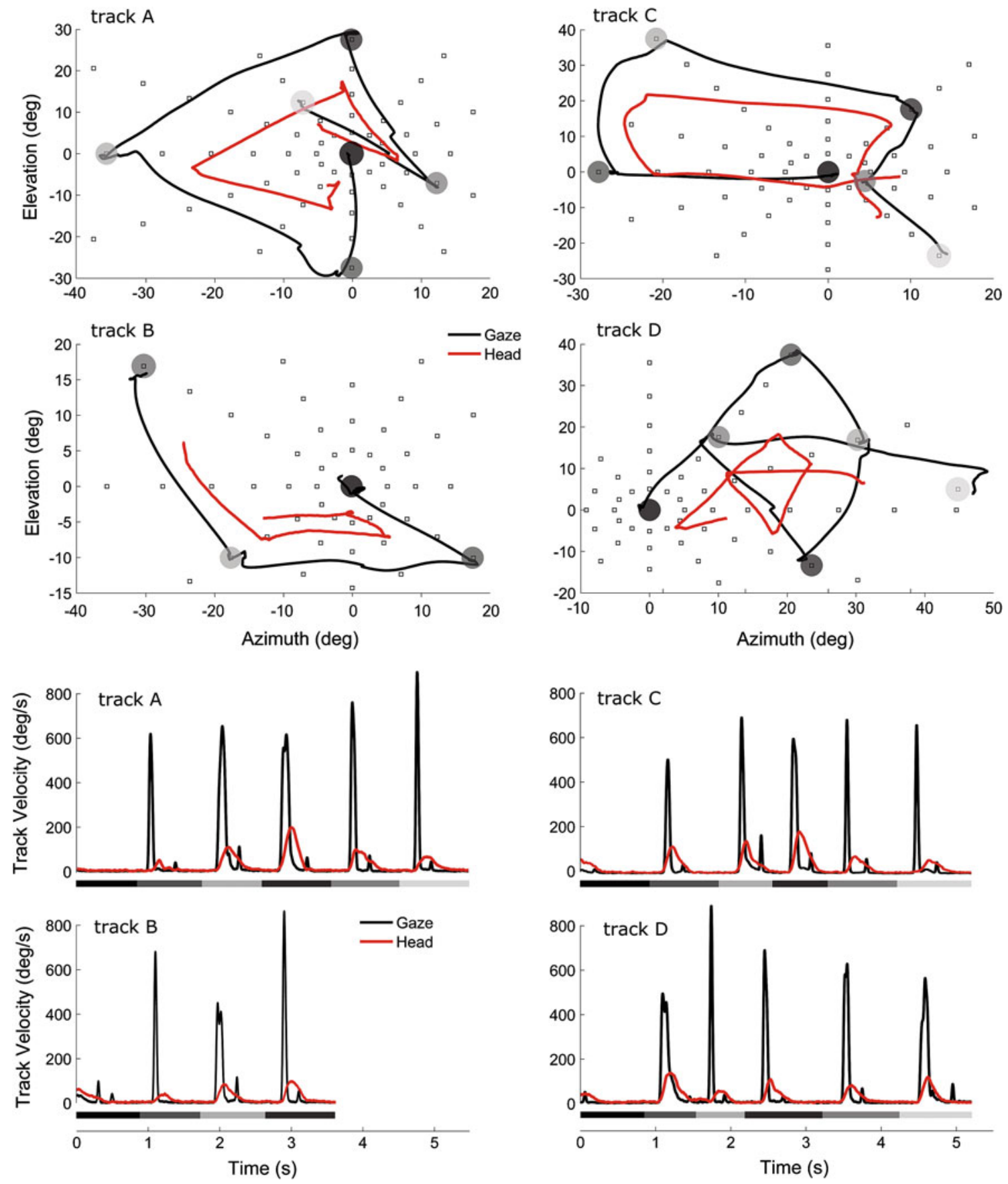

Fig. 10 Examples of calibrated gaze (black line) and head (red line) trajectories of monkey MA performing in four different trials (trajectories $\mathrm{A}-\mathrm{D}$ ) of the visual target following paradigm. The monkey gets rewarded when he detects the dimming of the final target (in lightest grey). Because the number of targets cannot be anticipated, the monkey has foveated all intervening targets (darker grey circles) as well. The darkest grey circle indicates the initial fixation point at $(0,0)^{\circ}$. Note that

eye-in-space and head-in-space follow very similar, but not identical, trajectories. The lower half of the figure shows the associated vectorial velocity profiles of gaze and head as function of time, as well as the stimulus presentation times. Despite substantial head movements, the peak head velocities (about $200 \%$ s) are much lower than the gaze velocities (up to $900^{\circ} / \mathrm{s}$ )

DMI assembly should be manufactured separately for each individual animal. However, a given assembly can be used virtually indefinitely, as it fits on the animal in exactly the

same way for every recording session, as demonstrated by Fig. 11. In this way, the acquisition of calibration data in the beginning of an animal's training results to be a cumulative 

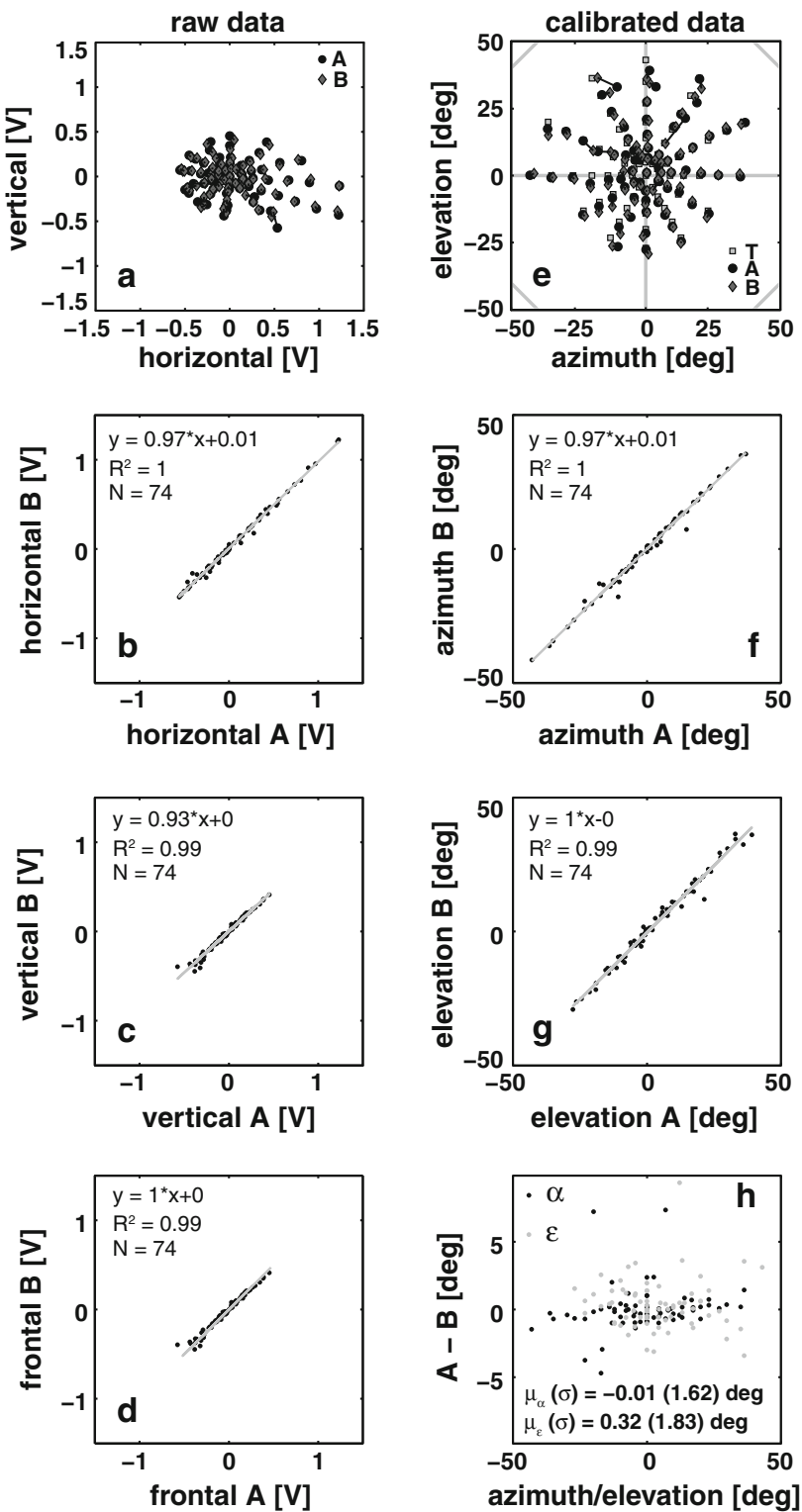

Fig. 11 Analysis of the stability of the DMI assembly. Left column raw data. Right column calibrated data. Data from set A (black circles) and $\mathrm{B}$ (dark grey diamonds) were collected on two consecutive days. a Raw endpoints of set A and B. Corresponding endpoints are connected with black lines. b Horizontal field set A as a function of horizontal field B (black circles). The grey line is a linear fit through the data. c Same as (b) for the vertical field. d Same as (b) for the frontal field. e Calibrated endpoints. Networks were trained on set A and used to calibrate both data sets. Target locations are shown as light grey squares. f Azimuth component of set B plotted against azimuth component of set A. The grey line is a linear fit through the data. $\mathbf{g}$ Same as (f) for elevation. h Difference between set A and B of the azimuth (black circles) and elevation (grey circles) components as function of location

process that yields better calibration results with increasing number of recording sessions. From this point onwards, the experimenter only has to quickly check the quality of the calibration at the beginning of a recording session, as is now done routinely in our lab.
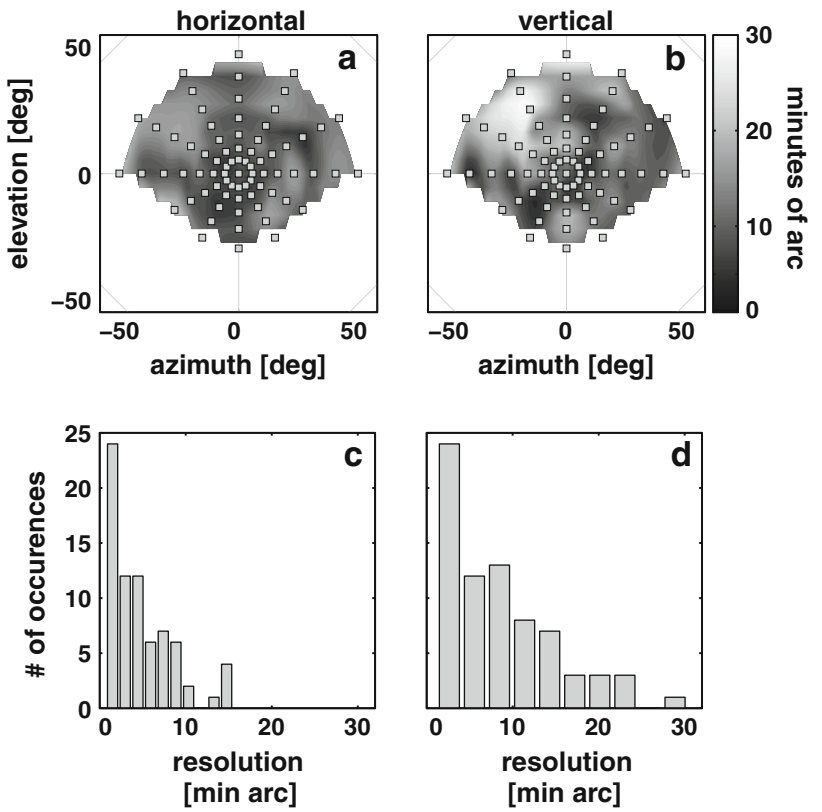

Fig. 12 Resolution of the head-unrestrained DMI method for the horizontal (a) and vertical (b) component in minutes of arc (shades of grey)

\section{Conclusions}

Studies employing animal subjects will directly benefit from the DMI method as presented here. Owing to the absence of vulnerable lead wires, the DMI method is more reliable. At the same time, the temporal and spatial resolution is comparable to the resolution of the SSC technique. The calibration routine presented here is easy to perform and straightforward to implement. We, therefore, propose that the DMI method is a valuable alternative to the classical SSC technique.

Acknowledgements We are grateful to Hans Kleijnen, Dick Heeren, Günter Windau, and Stijn Martens for their valuable technical assistance, and to Alex Hanssen and the central animal facility for excellent monkey care. This research was supported by a Marie Curie Early Stage Training Fellowship of the European Community's Sixth Framework Program (MEST-CT-2004-007825 PB), a VICI grant within the Earth and Life Sciences of NWO (ALW 865.05.003; AJVO, RFVDW, MMVW, TJVG), The Radboud University Medical Centre (DFS), and the Radboud University Nijmegen (AJVO).

Open Access This article is distributed under the terms of the Creative Commons Attribution Noncommercial License which permits any noncommercial use, distribution, and reproduction in any medium, provided the original author(s) and source are credited.

\section{References}

Allik J, Rauk M, Luuk A (1981) Control and sense of eye movement behind closed eyelids. Perception 10: 39-51

AUCAAC (2001) Policy statement: water restriction in rhesus behavior studies, A.U.a.C.A.A.C. The University of California, Davis 
Bos JE, Reulen JPH, Boersma HJ, Ditters BJ (1988) Theory of double magnetic induction (DMI) for measuring eye movements: correction for nonlinearity and simple calibration in two dimensions. IEEE Trans Biomed Eng 35:733-739

Bour LJ, Van Gisbergen JAM, Bruijns J, Ottes FP (1984) The double magnetic induction method for measuring eye movement-results in monkey and man. IEEE Trans Biomed Eng 31:419-427

Bremen P, Van der Willigen RF, Van Opstal AJ (2007a) Using double-magnetic induction to measure head-unrestrained gaze shifts. I. Theory and validation. J Neurosci Methods 160:75-84

Bremen P, Vander Willigen RF, Van Opstal AJ (2007b) Applying double magnetic induction to measure two-dimensional headunrestrained gaze shifts in human subjects. J Neurophysiol 98: 3759-3769

Collewijn H, Van der Mark F, Jansen TC (1975) Precise recording of human eye movements. Vision Res 15:447-450

Fuller JH (1992) Head movement propensity. Exp Brain Res 92:152164

Gander W, Golob GH, Strebel R (1994) Least squares fitting of circles and ellipses. BIT Numer Math 34:558-578

Goossens HHLM, Van Opstal AJ (1997) Human eye-head coordination in two dimensions under different sensorimotor conditions. Exp Brain Res 114:542-560

Hofman PM, Van Opstal AJ (1998) Spectro-temporal factors in two-dimensional human sound localization. J Acoust Soc Am 103:2634-2648

Irving EL, Zacher JE, Allison RS, Callender MG (2003) Effects of scleral search coil wear on visual function. Invest Ophthalmol Vis Sci 44:1933-1938

Judge SJ, Richmond BJ, Chu FC (1979) Implantation of magnetic search coils for measurement of eye position: an improved method. Vis Res 20:535-538
Krommenhoek KP, Van Opstal AJ, Van Gisbergen JAM (1996) An analysis of craniocentric and oculocentric coding stages in a neural network model of the saccadic system. Neural Netw 9: $1497-1511$

MacKay DJC (1992) Bayesian interpolation. Neural Comput 4:415447

Malpeli JG (1998) Measuring eye position with the double magnetic induction method. J Neurosci Methods 86:55-61

Murphy PJ, Duncan AL, Glennue AJ, Knox PC (2001) The effect of scleral coil lens wear on the eye. Br J Opthalmol 85:332-335

Ottes FP, Van Gisbergen JAM, Eggermont JJ (1987) Collicular involvement in a saccadic colour discrimination task. Exp Brain Res 66:465-478

Reulen JP, Bakker L (1982) The measurement of eye movement using double magnetic induction. IEEE Trans Biomed Eng 29:740-744

Robinson DA (1963) A method of measuring eye movement using a scleral search coil in a magnetic field. IEEE Trans Biomed Eng 10:137-145

Turner DA, Anderson IJ, Mason JC, Cox MG (1999) An algorithm for fitting an ellipsoid to data. http://www.scientificcommons.org/ 42871609. doi:10.1.1.36.2773

Van Gisbergen JA, Robinson DA, Gielen S (1981) A quantitative analysis of generation of saccadic eye movements by burst neurons. J Neurophysiol 45:417-442

Van Gisbergen JAM, Van Opstal AJ, Schoenmakers JJM (1985) Experimental test of two models for the generation of oblique saccades. Exp Brain Res 57:321-336

Van Wetter SMCI, Van Opstal AJ (2008) Experimental test of visuomotor updating models that explain perisaccadic mislocalisation. $\mathrm{J}$ Vis $8: 1-12$

Zwiers MP, Versnel H, Van Opstal AJ (2004) Involvement of monkey inferior colliculus in spatial hearing. J Neurosci 24:4145-4156 